\title{
Compound Gamma Representation for Modeling Travel Time Variability in a Traffic Network
}

\author{
Jiwon Kim \\ jiwon.kim@uq.edu.au \\ School of Civil Engineering, The University of Queensland \\ Brisbane St. Lucia, QLD 4072, Australia \\ Phone: +61 73346 3008; Fax: +61 733654599 \\ Hani S. Mahmassani* \\ masmah@northwestern.edu \\ Transportation Center, Northwestern University, \\ 600 Foster St., Evanston, IL 60208 \\ Phone: (847) 491-2276; Fax: (847) 491-3090
}

*corresponding author 


\title{
Compound Gamma Representation for Modeling Travel Time Variability in a Traffic Network
}

\author{
Jiwon Kim and Hani S. Mahmassani
}

\begin{abstract}
This paper proposes a compound probability distribution approach for capturing both vehicle-to-vehicle and day-to-day variability in modeling travel time reliability in a network. Starting from the observation that standard deviation and mean of distance-normalized travel time in a network are highly positively correlated and their relationship is well characterized by a linear function, this study assumes multiplicative error structures to describe data with such characteristics and derives a compound distribution to model travel delay per unit distance as a surrogate for travel time. The proposed GammaGamma model arises when (within-day) vehicle-to-vehicle travel delay per unit distance is distributed according to a Gamma distribution, with mean that itself fluctuates from day to day following another Gamma distribution. The study calibrates the model parameters and validates the underlying assumptions using both simulated and actual vehicle trajectory data. The Gamma-Gamma distribution shows good fits to travel delay observations when compared to the (simple) Gamma and Lognormal distributions. The main advantage of the Gamma-Gamma model is its ability to recognize different variability dimensions reflected in travel time data and clear physical meanings of its parameters in connection with vehicle-tovehicle and day-to-day variability. Based on the linearity assumption for the relationship between mean and standard deviation, two shape parameters of the Gamma-Gamma model are linked to the coefficient of variation of travel delay in vehicle-to-vehicle and day-to-day distributions, respectively, and can be directly estimated from the slope of the associated mean-standard deviation plots. An extension of the basic model form was also introduced to address potential deviations from this linearity assumption. The extended Gamma-Gamma model can account for time-of-day variations in mean-standard deviation relationships - such as hysteresis patterns observed in mean and day-to-day variation in travel time - and incorporate such dynamics in travel time distribution modeling. In summary, the model provides a systematic way of quantifying, comparing, and assessing different types of variability, which is important in understanding travel time characteristics and evaluating various transportation measures that affect reliability.
\end{abstract}

\section{KEYWORDS:}

Travel time reliability, Travel time variability, Compound probability distribution, Gamma-Gamma distribution, Network performance models, Network science. 


\section{INTRODUCTION}

Characterizing travel time variability using a mathematical model is a powerful means of understanding the statistical properties of travel time and quantifying the reliability performance of traffic networks. As a basis for deriving a wide range of reliability measures, various probability distributions have been proposed to describe the distribution of travel time. By far the most widely used are the Normal, Lognormal, Gamma, and Weibull distributions; many studies provide discussions and suggestions about the use of these models for fitting travel time data under different circumstances (Al-Deek and Emam, 2006; Arroyo and Kornhauser, 2005; Mazloumi et al., 2010; Polus, 1979; Pu, 2011; Rakha et al., 2006). Limitations of these traditional distributions have also been recognized and a number of alternatives have been explored. For instance, Susilawati et al. (2011) and Taylor et al. (Taylor and Susilawati, 2012) suggested the Burr Type XII distribution as an alternative statistical model for travel times with an upper long tail. Guo et al. (2010) proposed mixture distributions to describe multi-modality in travel time distributions that is often observed in the travel time data collected under multi-state (multi-regime) traffic conditions. Fosgerau and Fukuda (2012) used stable distributions to describe travel times, where the Normal and Cauchy distributions are special cases of this family. The study suggests that stable distributions can reproduce the empirical travel time distributions with high skewness and heavy tails.

Another line of studies have focused on directly modeling a measure of travel time variability as a function of other variables. Mazloumi et al. (2010), and Peer et al. (2012) investigate day-to-day variability in travel time data by performing regression analyses with standard deviation (SD) as the dependent variable to identify factors that affect travel time variability.

There has been growing interest in characterizing the relationships between reliability measures and the mean travel time, i.e., using the mean travel time as an explanatory variable for predicting travel time variability. Research under the Strategic Highway Research Program 2 (SHRP 2) (Cambridge Systematics, Inc. et al., 2010) developed analytical models for predicting reliability matrices such as $80^{\text {th }}$ and $95^{\text {th }}$ percentile travel time indices and on-time arrival probability as a function of the mean travel time index. Mahmassani et al. (2012a) explored various functional forms to characterize the relationship between the SD of travel time per unit distance across vehicles and its mean and proposed a linear relation as a robust method for predicting the SD. That approach was further explored in (Mahmassani et al., 2013a) and integrated in a methodology for incorporating reliability in dynamic network equilibrium models to evaluate user responses to pricing (Jiang et al., 2011).

Travel time variability can be defined at varying levels from different perspectives, namely, dayto-day variability, within-day or period-to-period variability and vehicle-to-vehicle variability (Noland and Polak, 2002; Yildirimoglu et al., 2013). Most previous studies have focused on only one particular type of variability; the linkages between different variability dimensions have not been accounted for in probability distributions and predictive models discussed above. As detailed traffic data such as individual vehicle trajectories are becoming increasingly available (Mahmassani et al., 2012c), it is important to recognize different types of variability reflected in the data in order to fully characterize travel time reliability and accurately assess the effects of various transportation operational measures on each type of variability.

This study addresses this need by proposing a compound distribution representation of travel delay per unit distance that enables us to capture both vehicle-to-vehicle and day-to-day variability. The model, termed Gamma-Gamma distribution, arises as the product of two Gamma distributions. The use of the Gamma-Gamma distribution in travel time reliability modeling was first introduced in (Kim, 2014) 
and its application to the characterization of vehicle-to-vehicle and day-to-day travel time variability was presented in (Kim and Mahmassani, 2014) using simulated trajectories. The present paper develops the methodological foundations of the Gamma-Gamma model, including its formal derivation, and validation using both simulated and actual vehicle trajectory data, and introduces a generalized form that may be applicable to non-stationary conditions. The basic derivation of the Gamma-Gamma model is motivated by a specific characteristic observed in travel time data, namely, a strong positive correlation between mean and SD. Based on the observation that such characteristics are present in both vehicle-to-vehicle and day-to-day travel time distributions, we express the SD of each component distribution as a function of its mean and link the two relations to form a compound probability density function (PDF) that reflects the overall travel time variability. The proposed model is mathematically tractable in that its PDF is available in closed form, and its parameters have clear physical meaning; that is the two shape parameters represent the inverse of the square of coefficient of variation $(\mathrm{CV})$ of vehicle-to-vehicle and day-to-day distributions, respectively. As such, one can directly obtain the model parameters by estimating mean and SD statistics from travel time data. Its ability to recognize and decompose different variability dimensions provides a systematic way of performing the comparative evaluation of policies or projects that affect travel time reliability. Moreover, the model can be used as a useful tool for simulation-based reliability analyses as the straightforward interpretation of model parameters allows one to easily adjust and change parameter values to examine travel time distributions under different types and levels of variability. It is, however, noted that the present paper focuses on the statistical representation of the travel time distribution using the aforementioned concepts; interpretation and application of the representation captured by the proposed model for actual policy analysis is outside the scope of the paper.

The rest of this paper is organized as follows. Section 2 presents approaches to characterizing vehicle-to-vehicle and day-to-day travel delay distributions based on relationships between mean and SD. Section 3 presents the detailed model derivation, properties of the Gamma-Gamma distribution, and some extension of the basic model form. Section 4 and Section 5 describe results for model validation and parameter estimation conducted using simulated and actual vehicle trajectory data, respectively. Section 6 presents applications of the Gamma-Gamma distribution, and Section 7 provides summary and conclusion. 


\section{CHARACTERIZING DIFFERENT COMPONENTS OF TRAVEL TIME VARIABILITY}

\subsection{Vehicle-to-Vehicle Variability: Relation between Mean and Standard Deviation of Travel Delay per Unit Distance}

Vehicle-to-vehicle variability refers to travel time variation between drivers who depart within the same time slot. Excluding the effect of unpredictable exogenous events, which are typically modeled through scenario-based approaches (Kim et al., 2013b), the major sources of the variability considered here include interactions between vehicles and heterogeneity in driving behavior. Previous studies have shown that there exists a strong positive correlation between mean and SD of travel time across vehicles, i.e., the vehicle-to-vehicle variability increases with the level of congestion. Jones et al. (1989) investigated commuting data in Austin, Texas, USA at the link level and found that there is a linear relationship between the mean and SD of trip time per mile, where each sample point is obtained from data collected during a 5-minute observation period. Recently Mahmassani et al. (2013a, 2012a) confirmed this linear relationship using both simulated and actual vehicle trajectory data and found it robust across different aggregation levels (e.g., network, origin-destination, path and link) and different scales of networks. They emphasize the use of distance-normalized travel time "to exclude the source of variability coming from trip distance and focus on the travel time variability caused by variation of speed." They express the relationship between mean and SD of distance-normalized travel time using the following simple linear function (Mahmassani et al., 2013a):

$$
\sigma_{\tau}=\theta_{1}+\theta_{2} \mu_{\tau}+\varepsilon
$$

where

$$
\begin{array}{ll}
\tau & : \text { travel time per unit distance } \\
\mu_{\tau}, \sigma_{\tau} & : \text { mean and SD of } \tau, \text { respectively } \\
\theta_{1}, \theta_{2} & : \text { coefficients to be estimated. } \\
\varepsilon & : \text { random error }
\end{array}
$$

The slope term $\left(\theta_{2}\right)$ represents the average increase in the SD for each one-unit increase in the mean, i.e., a greater $\theta_{2}$ means greater variability and less reliability. The negative ratio between $\theta_{1}$ and $\theta_{2}$ $\left(-\theta_{1} / \theta_{2}\right)$, i.e., the $\mathrm{x}$-intercept, represents the minimum travel time per unit distance, which is the reciprocal of the free flow speed.

Suppose we have a random variable $x$ defined as $x=\tau-\left(-\theta_{1} / \theta_{2}\right)$. The mean and SD of $x$ are $\mu_{x}=\mu_{\tau}+\theta_{1} / \theta_{2}$ and $\sigma_{x}=\sigma_{\tau}$, respectively. Substituting $\mu_{\tau}=\mu_{x}-\theta_{1} / \theta_{2}$ and $\sigma_{\tau}=\sigma_{x}$ into Eq. (1) yields $\sigma_{x}=\theta_{2} \mu_{x}+\varepsilon$, where $\theta_{2}$ now becomes the coefficient of variation (CV) of $x$ if random error $\varepsilon$ goes to zero. With $\varepsilon$ being fairly small, this implies that the $\mathrm{CV}$ of $x$ is constant over the range of its mean and the SD of $x$ is approximately proportional to the mean level. In our context, variable $x$ can be viewed as travel delay per unit distance, which is obtained by subtracting the minimum travel time per unit distance $\left(-\theta_{1} / \theta_{2}\right)$ from individual travel time per unit distance $(\tau)$. Working with travel delay rather than travel time allows us to use the direct proportionality between mean and SD (or constant CV), which is the key property that allows us to derive an analytically tractable compound probability distribution model used in this study, and thus we will use travel delay $x$ as a surrogate for travel time for the subsequent analysis. 
Travel time and travel delay will refer to travel time per unit distance and travel delay per unit distance, respectively, for the rest of the paper.

Let $X_{t, n}$ denote a random variable representing the travel delay per unit distance of an arbitrary vehicle at time interval $t$ on day $n$ and $x_{t, n}$ denote the value of $X_{t, n}$. For time interval $t=1, \cdots, T$ and day $n=1, \cdots, N$, the mean and SD of $X_{t, n}$ across vehicles, denoted by $\mu_{X_{t, n}}$ and $\sigma_{X_{t, n}}$, respectively, are estimated by:

$$
\hat{\mu}_{X_{t, n}}=\frac{1}{v_{t, n}} \sum_{i=1}^{v_{t, n}} x_{t, n, i}
$$

and

$$
\hat{\sigma}_{X_{t, n}}=\sqrt{\frac{1}{v_{t, n}} \sum_{i=1}^{v_{t, n}} x_{t, n, i}^{2}-\left(\hat{\mu}_{X_{t, n}}\right)^{2}}
$$

where

$$
\begin{array}{ll}
n & : \text { day index } \\
t & : \text { time-of-day index } \\
i & : \text { vehicle index }
\end{array}
$$

$\mu_{X_{t, n}}, \sigma_{X_{t, n}} \quad$ : vehicle-to-vehicle mean and SD of travel delay $X_{t, n}$ across vehicle $i$ within $t$ and $n$

$$
\begin{array}{ll}
v_{t, n} & : \text { number of vehicles observed within } t \text { and } n \\
x_{t, n, i} & \text { : value of } X_{t, n} \text { for the } i^{\text {th }} \text { vehicle within } t \text { and } n
\end{array}
$$

The hat $(\wedge)$ indicates an estimator.

Based on the discussion above, we express the relationship between the vehicle-to-vehicle mean and SD of $X_{t, n}$ using the following linear equation:

$$
\sigma_{X_{t, n}}=\alpha \mu_{X_{t, n}}+\varepsilon,
$$

where $\varepsilon$ represents a random error. The slope parameter $\alpha$ corresponds $\theta_{2}$ in Eq. (1) and can be interpreted as the $\mathrm{CV}$ of $X_{t, n}$ if $\varepsilon$ goes to zero.

\subsection{Day-to-Day Variability: Relation between Mean and Standard Deviation of Mean Travel Delay per Unit Distance}

Day-to-day variability concerns how travel time along a particular route in a network at a given departure time interval varies over different days. The major sources of the variability include: external events such as incidents, work-zone, and weather conditions; variations in day-to-day demand levels; and traffic control devices (Kim et al., 2013b; Mahmassani et al., 2013b).

Despite the conceptual difference between vehicle-to-vehicle variability (variability over space) and day-to-day variability (variability over time), there seems to be a connection between the two in terms of the relationship between mean and SD. Several previous studies that investigated daily travel time data have reported the existence of a strong positive correlation between day-to-day variability and the 
congestion level. Herman and Lam (1974) developed a theory that the SD is proportional to the square root of the mean travel time, based on travel time data collected by different drivers on their normal work journeys along different routes over successive days. Richardson and Taylor (1978) later extended the theory of Herman and Lam (1974) using an alternative method of data collection to isolate day-to-day variability from other sources of variation such as different driver and route characteristics. May et al. (1989) conducted a longitudinal study to collect daily travel time data and found that the relationship between SD and mean journey time was approximated equally well by a linear or square-root function. Such relations are also found in public transport trips. Taylor (1982) studied the variability of daily travel time on two public transport modes in Paris, France and confirmed the relation found in the earlier work (Herman and Lam, 1974; Richardson and Taylor, 1978). Mazloumi et al. (2010) examined day-to-day bus travel time data in Melbourne, Australia and showed that the SD increases linearly with the mean.

In addition to an increasing relation between mean and variation in travel times, several studies have also noted that there exists a hysteresis pattern in the relationship between day-to-day travel time variation and its mean, i.e., day-to-day travel time variation during the onset of congestion is higher or lower than that during the congestion offset, with the same mean level. Fosgerau (2010) provided a theoretical explanation for counter-clockwise hysteresis patterns observed at congested bottlenecks. Considering a recurrent bottleneck that has the first-in-first-out (FIFO) queueing discipline and random capacity and demand, the study expresses the mean and variance of time in the queue as functions of time and shows that the time derivatives of the mean and variance are related to each other as to form a loop. Gayah et al. (2013) provided a theoretical explanation for counter-clockwise hysteresis loops in networkwide travel times. Using the network traffic flow theory, the study shows that the clockwise hysteresis loops in the Macroscopic Fundamental Diagram (MFD) in urban traffic imply counter-clockwise loops in the relationship between day-to-day mean and variance of travel time. Yildirimoglu et al. (2013) investigated both clockwise or counter-clockwise hysteresis loops observed in freeway route travel time data and empirically analyzed factors that affect the shape and direction of the loops.

Based on the findings in the literature, we make the assumption that a linear relationship also exists between the mean and SD estimated from day-to-day travel time distributions. Unlike the vehicleto-vehicle variability case, the approach to measuring day-to-day variability greatly varies from study to study in the literature. To gain further empirical evidence to support our assumption, we investigated freeway travel time data obtained from the Caltrans Performance Measurement System (PeMS), which collects historical and real-time traffic data from freeways in California, USA. The PeMS site provides travel time data for selected routes aggregated over 5-minute intervals. The system computes the travel time on a route by obtaining the speed of each segment based on the time a vehicle arrives at that segment to reproduce experienced travel times ("PeMS," n.d.). For the analysis, we selected two routes with different lengths: (i) a 20.7-mile section of I-880S in the San Francisco Bay Area and (ii) a 4.9-mile section of I-405N in Irvine. The 5-minute travel time data were collected for the entire year of 2012. After excluding weekends and holidays, we obtained data for a total of 251 days. For each 5-minute interval, we computed the mean and SD based on 251 daily observations of travel time per mile. FIGURE 1 presents mean-SD plots for the morning period (6AM - 12PM) and afternoon period (12PM - 9PM) for each route. Data points are connected in the order of their succession in time to examine successive changes in the mean and SD, where hysteresis loops are observed as in the literature (Fosgerau, 2010; Fosgerau and Fukuda, 2012; Gayah et al., 2013; Yildirimoglu et al., 2013). The overall trend of the data points in each plot, however, shows a linearly or near-linearly increasing pattern and the coefficient of determination $\left(R^{2}\right)$ values are found to be significant. 


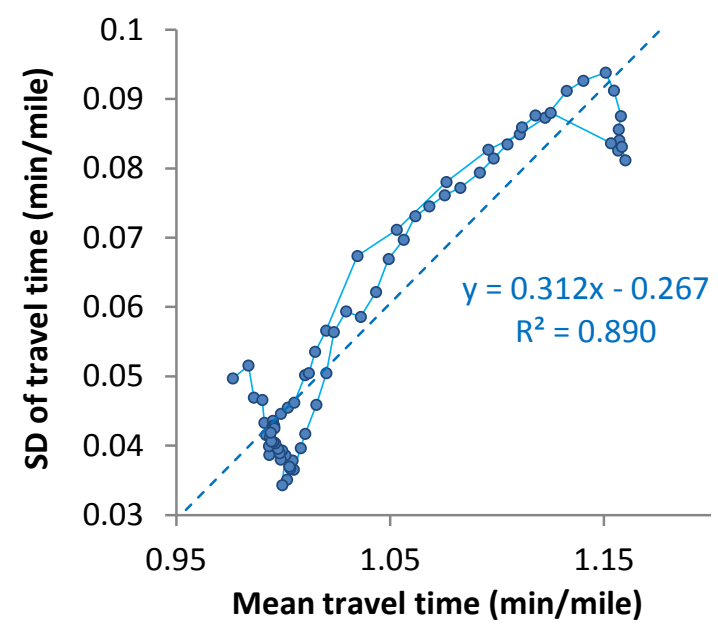

(a)

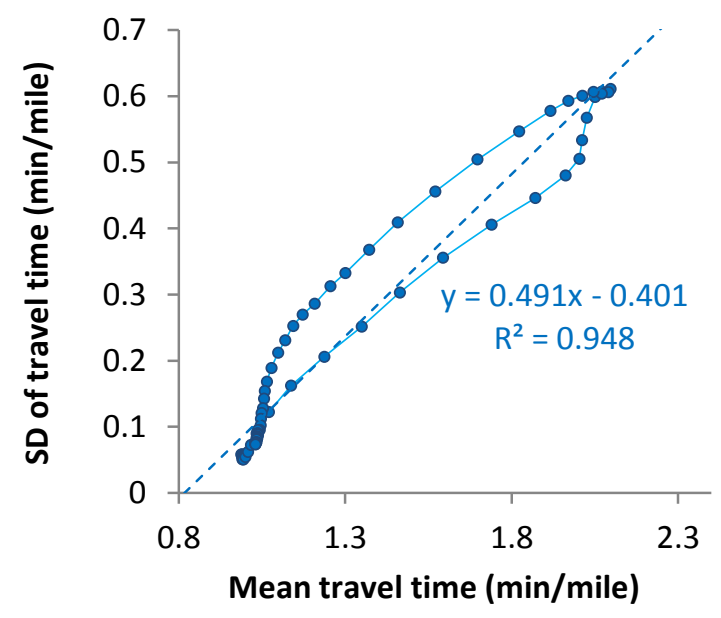

(c)

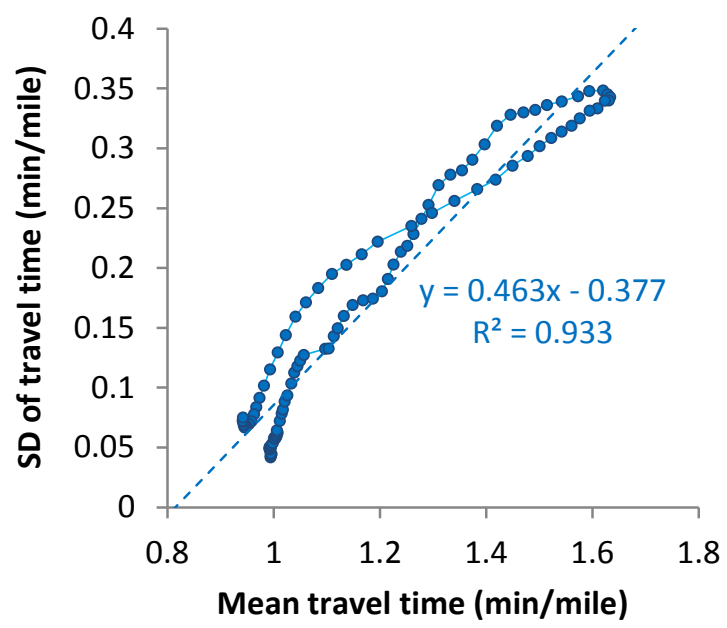

(b)

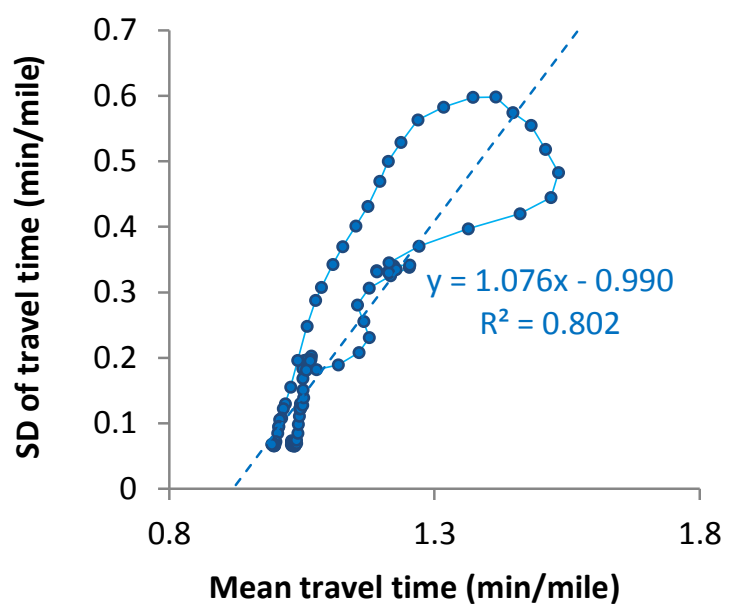

(d)

FIGURE 1 Relation between mean and SD of travel time per unit distance in its day-to-day distribution; data are from freeways in California, USA: (a) I-880S (6AM - 12PM), (b) I-880S (12PM - 9PM), (c) I-405N (6AM - 12PM) and (d) I-405N (12PM - 9PM).

Given the assumption of linear relationship between mean and SD of day-to-day travel time distribution, we derive the relationship between mean and SD of day-to-day travel delay in a similar manner to the case of vehicle-to-vehicle travel delay distribution. Let $Y_{t}$ denote a random variable representing day-today travel delay per unit distance at time interval $t$, which is defined as the mean of individual travel delay per unit distance across vehicles within $t$ on an arbitrary day, and $y_{t}$ denote the value of $Y_{t}$. Given $N$ days $(n=1, \cdots, N)$, the mean and SD of $Y_{t}$ across days, denoted by $\mu_{t}$ and $\sigma_{t}$, respectively, are estimated using the following equations:

$$
\hat{\mu}_{t}=\frac{1}{N} \sum_{n=1}^{N} y_{t, n}
$$


and

$$
\hat{\sigma}_{t}=\sqrt{\frac{1}{N} \sum_{n=1}^{N} y_{t, n}^{2}-\left(\hat{\mu}_{t}\right)^{2}},
$$

where

$\mu_{t}, \sigma_{t}:$ day-to-day mean and $\mathrm{SD}$ of travel delay at $t, Y_{t}$, across days

$N \quad$ : total number of days

$y_{t, n} \quad$ : value of $Y_{t}$ on day $n$.

From the definition of $Y_{t}$, it is noted that $y_{t, n}$ in Eqs. (5) and (6) represents the mean estimate of $X_{t, n}$, $\hat{\mu}_{X_{t, n}}$ in Eq. (2), i.e., $y_{t, n}=\hat{\mu}_{X_{t, n}}=\left(1 / v_{t, n}\right) \sum_{i=1}^{v_{t, n}} x_{t, n, i}$.

Based on the empirical evidence and discussions provided above, we express the relationship between the day-to-day mean and SD of $Y_{t}$ using the following linear equation:

$$
\sigma_{t}=\beta \mu_{t}+\xi,
$$

where $\xi$ is a random error. Similar to $\alpha$ in Eq. (4), slope parameter $\beta$ can be interpreted as the CV of mean travel delay $Y_{t}$ if $\xi$ is zero.

To summarize, FIGURE 2 shows the relations among variables defined so far. The distribution plot in each cell $(t, n)$ represents vehicle-to-vehicle distribution of travel delay per unit distance $X_{t, n}$; the distribution plot across $n$ at each $t$ represents day-to-day distribution of $Y_{t}$, i.e., the distribution of the mean of $X_{t, n}$. The distribution plot at the bottom of each $t$ represents the overall distribution of individual delay $X_{t}$, which aggregates vehicle-to-vehicle distributions over all $N$ days. The distribution of $X_{t}$ reflects both vehicle-to-vehicle and day-to-day variability and, thus, $X_{t}$ becomes the main variable that this study focuses on modeling using a compound representation. 


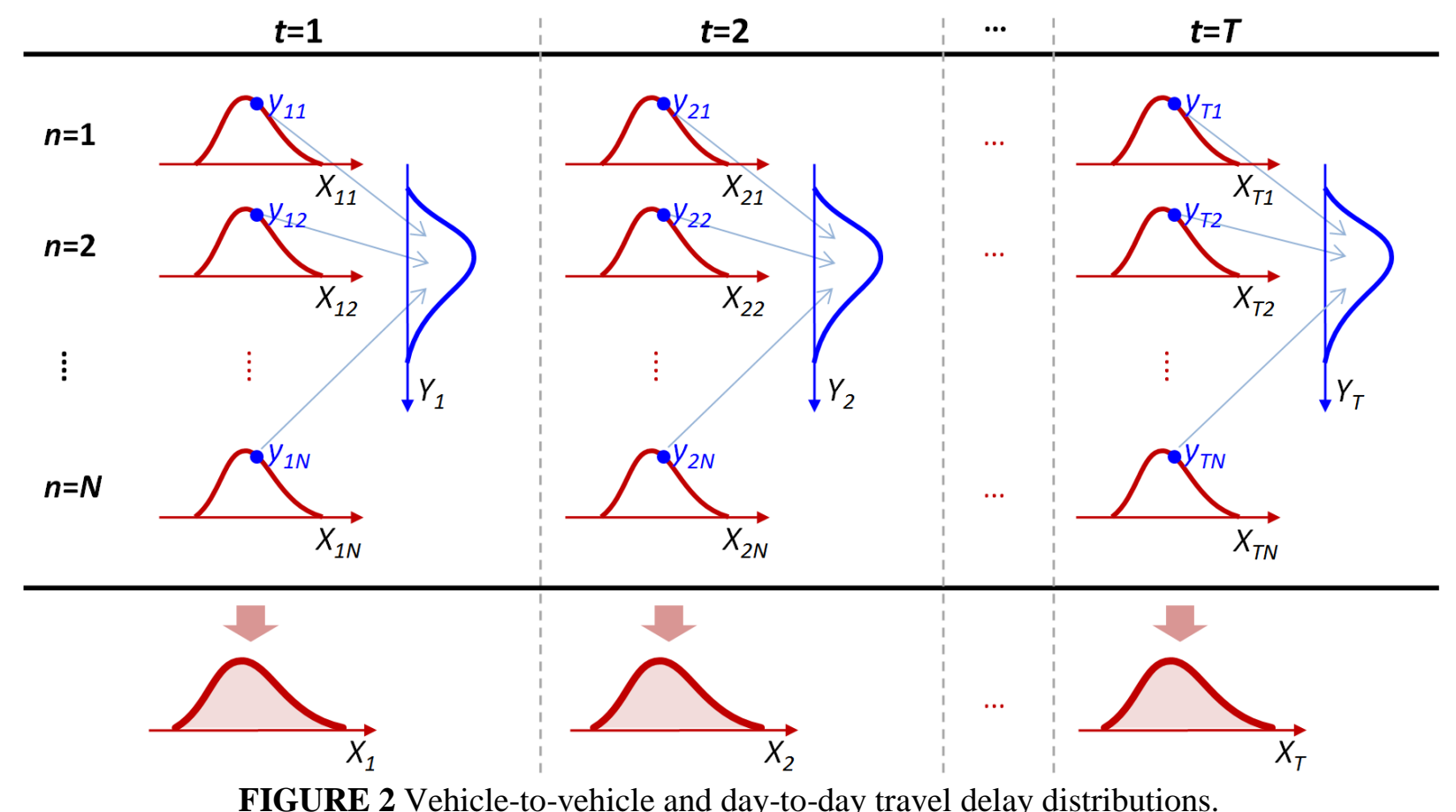

For now, we will assume that error terms $\varepsilon$ and $\xi$ in Eqs. (4) and (7) can be ignored, indicating that the $\mathrm{CVs}$ of $X_{t, n}$ and $Y_{t}$ are constant - corresponding to $\alpha$ and $\beta$, respectively - within given time and day boundary $(t=1, \cdots, T) \times(n=1, \cdots, N)$. Later, we will discuss the cases where the constant CV assumption does not hold and present an extended model that can address such deviations. When a random variable exhibits a constant $\mathrm{CV}$, a multiplicative error model is often used to account for this characteristic. In the next section, we derive multiplicative error models for $X_{t, n}$ and $Y_{t}$ based on these properties.

\section{MODELING TRAVEL DELAY USING A COMPOUND DISTRIBUTION}

\subsection{Multiplicative Error Model Based on Gamma distribution}

Classical linear models use a normal additive error structure, i.e., $z=\mu_{z}+\zeta$, where $z$ is a random response variable, $\mu_{z}$ is the mean of $z$, and $\zeta$ is a normally distributed random error with zero mean. In this specification, the variance of $z$ is assumed to be constant over the entire range of $\mu_{z}$. When the SD of $z$ increases proportionally to $\mu_{z}$, however, a multiplicative error structure, i.e., $z=\mu_{z} \zeta$ is a more appropriate representation, where $\zeta$ is a random error with mean 1 and constant variance $\sigma_{\zeta}^{2}$. This type of multiplicative error structure arises in many applications such as financial econometrics (Engle, 2002; Engle and Gallo, 2006) and regression analysis using generalized linear models (MacCullagh and Nelder, 1989). The above specification leads to $E[z]=\mu_{z}$ and $S D[z]=\sigma_{\zeta} \mu_{z}$, i.e., the $\mathrm{SD}$ of $z$ is proportional to its mean; and parameter $\sigma_{\zeta}$ determines a proportionality factor. For non-negative $z$, the error term can be specified by various (non-negative) parametric distributions such as Gamma, Lognormal, Weibull, and 
Inverse-Gamma as discussed in Brownlee et al. (2011). In this study, the Gamma distribution is used because of its mathematical tractability and ability to properly describe travel time data.

Applying this to our problem, we express the individual travel delay per unit distance at time interval $t$ on day $n, X_{t, n}$, using the following model:

$$
X_{t, n}=\mu_{X_{t, n}} \gamma,
$$

where $\mu_{X_{t, n}}$ is the vehicle-to-vehicle mean travel delay representing the daily congestion level at $t$ on given day $n$ and $\gamma$ is a random error that represents vehicle-to-vehicle variations in $X_{t, n}$ around its mean $\mu_{X_{t, n}}$. The error $\gamma$ is assumed to be independent and identically distributed (i.i.d.) across $t$ and $n$, following a Gamma distribution with shape parameter $\pi$ and unit mean (or scale parameter $1 / \pi$ ) denoted by $\gamma \sim \operatorname{Gamma}(\pi, 1 / \pi)$. Then, $X_{t, n}$ follows a Gamma distribution with shape parameter $\pi$ and mean $\mu_{X_{t, n}}$, denoted by $X_{t, n} \sim \operatorname{Gamma}\left(\pi, \mu_{X_{t, n}} / \pi\right)$.

Next, we specify the behavior of $Y_{t}$, daily congestion level (i.e., mean travel delay per unit distance) at time interval $t$, using the following model:

$$
Y_{t}=\mu_{t} \eta
$$

where $\mu_{t}$ is the day-to-day mean of $Y_{t}$ and $\eta$ is a random error that represents day-to-day fluctuations in $Y_{t}$ around its mean $\mu_{t}$. The error $\eta$ is assumed to be i.i.d across $t$, following a Gamma distribution with shape parameter $\phi$ and unit mean, i.e., $\eta \sim \operatorname{Gamma}(\phi, 1 / \phi)$, thereby letting $Y_{t} \sim \operatorname{Gamma}\left(\phi, \mu_{t} / \phi\right)$.

TABLE 1 summarizes detailed properties of these two models. Rows (a) through (c) describe the response variables, model structures, and error specifications, respectively; and row (d) presents the statistics of the response variables. The mean and variance of $X_{t, n}$ across vehicles are given by $\mu_{X_{t, n}}$ and $\mu_{X_{t, n}}^{2} / \pi$, respectively, and the mean and variance of $Y_{t}$ across days are given by $\mu_{t}$ and $\mu_{t}^{2} / \phi$, respectively. The CV of $X_{t, n}$, which corresponds to the slope coefficient $\alpha$ in Eq. (4), is expressed as $1 / \sqrt{\pi}$; and, similarly, that of $Y_{t}, \beta$ in Eq. (7), is given by $1 / \sqrt{\phi}$. As a result, the skewness of $X_{t, n}$, defined as $2 / \sqrt{\pi}$, corresponds $2 \alpha$ and that of $Y_{t}$, defined as $2 / \sqrt{\phi}$, corresponds to $2 \beta$.

Recognizing that $\mu_{X_{t, n}}$ in Eq. (8) corresponds to $Y_{t}$ on day $n$ in Eq. (9), we can view $\mu_{X_{t, n}}$ as a random variable taking values of $Y_{t}$. By substituting $Y_{t}$ in Eq. (9) for $\mu_{X_{t, n}}$ in Eq. (8), we obtain the form $X_{t}=Y_{t} \gamma$, where the response variable $X_{t}$ represents individual travel delay at $t$ fluctuating across both vehicles and days. This form indicates that $X_{t}$ is expressed as the product of two Gamma-distributed random variables $Y_{t}$ and $\gamma$. In the next section, we show that there is a closed form expression for the probability density function (PDF) of this product distribution and discuss its use in the context of travel time distribution modeling. 
TABLE 1 Multiplicative Error Model Specification for Travel Delay per Unit Distance $X_{t, n}$ and Mean Travel Delay per Unit Distance $Y_{t}$

\begin{tabular}{|c|c|c|c|}
\hline & & $\begin{array}{l}\text { Modeling vehicle-to-vehicle } \\
\text { travel delay distribution }\end{array}$ & $\begin{array}{c}\text { Modeling day-to-day } \\
\text { mean travel delay distribution }\end{array}$ \\
\hline (a) & Response variable & $\begin{array}{c}\text { Individual travel delay per unit } \\
\text { distance at time-of-day } t \text { on day } n \text { : } \\
\qquad X_{t, n}\end{array}$ & $\begin{array}{l}\text { Mean travel delay per unit distance } \\
\text { at time-of-day } t \text { : } \\
Y_{t}\end{array}$ \\
\hline (b) & $\begin{array}{l}\text { Multiplicative } \\
\text { error model }\end{array}$ & $X_{t, n}=\mu_{X_{t, n}} \gamma$ & $Y_{t}=\mu_{t} \eta$ \\
\hline (c) & Error specification & $\begin{array}{c}\gamma \sim \operatorname{Gamma}(\pi, 1 / \pi) \\
E[\gamma]=\pi / \pi=1 \\
\operatorname{Var}[\gamma]=\pi / \pi^{2}=1 / \pi \\
C V[\gamma]=1 / \sqrt{\pi}\end{array}$ & $\begin{array}{c}\eta \sim \operatorname{Gamma}(\phi, 1 / \phi) \\
E[\eta]=\phi / \phi=1 \\
\operatorname{Var}[\eta]=\phi / \phi^{2}=1 / \phi \\
C V[\eta]=1 / \sqrt{\phi}\end{array}$ \\
\hline (d) & $\begin{array}{l}\text { Distribution of } \\
\text { response variable }\end{array}$ & $\begin{array}{c}X_{t, n} \sim \operatorname{Gamma}\left(\pi, \mu_{X_{t, n}} / \pi\right) \\
E\left[X_{t, n}\right]=\pi \times\left(\mu_{X_{t, n}} / \pi\right)=\mu_{X_{t, n}} \\
\operatorname{Var}\left[X_{t, n}\right]=\pi \times\left(\mu_{X_{t, n}} / \pi\right)^{2}=\mu_{X_{t, n}}^{2} / \pi \\
\operatorname{CV}\left[X_{t, n}\right]=1 / \sqrt{\pi} \\
\operatorname{Skew}\left[X_{t, n}\right]=2 / \sqrt{\pi}\end{array}$ & $\begin{array}{c}Y_{t} \sim \operatorname{Gamma}\left(\phi, \mu_{t} / \phi\right) \\
E\left[Y_{t}\right]=\phi \times\left(\mu_{t} / \phi\right)=\mu_{t} \\
\operatorname{Var}\left[Y_{t}\right]=\phi \times\left(\mu_{t} / \phi\right)^{2}=\mu_{t}^{2} / \phi \\
\operatorname{CV}\left[Y_{t}\right]=1 / \sqrt{\phi} \\
\operatorname{Skew}\left[Y_{t}\right]=2 / \sqrt{\phi}\end{array}$ \\
\hline (e) & $\begin{array}{l}\text { Compound } \\
\text { distribution }\end{array}$ & \multicolumn{2}{|c|}{$\begin{array}{l}\text { Overall distribution of individual travel delay } X_{t} \text { across vehicles and days: } \\
\left.\qquad X_{t} \sim \operatorname{GammaGammd} \pi, \phi, \mu_{t}\right) \\
\text { where } X_{t}=Y_{t} \gamma, \gamma \sim \operatorname{Gamma}(\pi, 1 / \pi) \text { and } Y_{t} \sim \operatorname{Gamma}\left(\phi, \mu_{t} / \phi\right)\end{array}$} \\
\hline
\end{tabular}

\subsection{Compound Representation of Travel Delay: Gamma-Gamma Distribution}

The form $X_{t}=Y_{t} \gamma$ along with our distributional assumptions indicates that $X_{t}$ is distributed according to a product distribution of two independent Gamma random variables $Y_{t}$ and $\gamma$. Since $Y_{t}$ represents the mean of $X_{t}$, the distribution of $X_{t}$ is also characterized as a compound distribution in which $X_{t}$ is distributed according to a Gamma distribution with mean $Y_{t}$, with the mean itself being a random variable following another Gamma distribution. The first Gamma distribution reflects vehicle-to-vehicle variations in individual travel delay under a particular congestion level and the second Gamma distribution reflects day-to-day fluctuations in the congestion level at a given time interval. The random nature of the daily congestion level makes it necessary to use such a compound representation or doubly stochastic model to correctly describe the overall travel delay distribution that captures both the temporal (day-to-day) and the spatial (vehicle-to-vehicle) variations in travel delay.

When two Gamma random variables are compounded in this way-the mean of a Gamma distribution is distributed according to another Gamma distribution-, a closed form expression exists for the PDF of the compound distribution and we present its derivation below. 
The PDF of $Y_{t}$ under $Y_{t} \sim \operatorname{Gamma}\left(\phi, \mu_{t} / \phi\right)$ is given by:

$$
f_{Y_{t}}\left(y_{t}\right)=\left(\frac{\phi}{\mu_{t}}\right)^{\phi} \frac{1}{\Gamma(\phi)} y_{t}^{\phi-1} e^{-y_{t}\left(\frac{\phi}{\mu_{t}}\right)},
$$

where $\Gamma(\cdot)$ is the Gamma function. The conditional PDF of $X_{t}$ given realization $y_{t}$ of $Y_{t}$ is the Gamma distribution from $X_{t, n} \sim \operatorname{Gamma}\left(\pi, \mu_{X_{t, n}} / \pi\right)$ and is written as:

$$
f_{X_{t} \mid Y_{t}}\left(x_{t} \mid Y_{t}=y_{t}\right)=\left(\frac{\pi}{y_{t}}\right)^{\pi} \frac{1}{\Gamma(\pi)} x_{t}^{\pi-1} e^{-x_{t}\left(\frac{\pi}{y_{t}}\right)} \text {. }
$$

The unconditional PDF of $X_{t}$ is then obtained by integrating $f_{X_{t} \mid Y_{t}}\left(x_{t} \mid y_{t}\right)$ over all possible values of $Y_{t}$ as follows:

$$
f_{X_{t}}\left(x_{t}\right)=\int_{0}^{\infty} f_{X_{t} \mid Y_{t}}\left(x_{t} \mid y_{t}\right) f_{Y_{t}}\left(y_{t}\right) d y_{t}
$$

By substituting Eqs. (10) and (11) into Eq. (12), we obtain the following intermediate form:

$$
f_{X_{t}}\left(x_{t}\right)=\frac{\pi^{\pi} x_{t}^{\pi-1}}{\Gamma(\pi) \Gamma(\phi)}\left(\frac{\phi}{\mu_{t}}\right)^{\phi} \int_{0}^{\infty} y_{t}^{\phi-\pi-1} e^{-\frac{\phi y_{t}}{\mu_{t}}-\frac{\pi x_{t}}{y_{t}}} d y_{t} .
$$

Setting $u=\phi y_{t} / \mu_{t}$ (i.e., $\left.d y_{t}=\left(\mu_{t} / \phi\right) d u\right)$ and rearranging Eq. (13) yields

$$
f_{X_{t}}\left(x_{t}\right)=\frac{1}{\Gamma(\pi) \Gamma(\phi)} \frac{1}{x_{t}}\left(\frac{\pi \phi x_{t}}{\mu_{t}}\right)^{\pi} \int_{0}^{\infty} u^{(\phi-\pi)-1} e^{-\left(\frac{\pi \phi x_{t}}{\mu_{t}}\right) \frac{1}{u}-u} d u .
$$

The integral part in Eq. (14) can be expressed in terms of the modified Bessel function of the second kind $K_{v}(z)$ using the following formula (Gradshteyn et al., 2007, Equation 3.471.9; Shankar, 2004):

$$
\int_{0}^{\infty} u^{v-1} e^{-\frac{\varphi}{u}-\rho u} d u=2\left(\frac{\varphi}{\rho}\right)^{\frac{v}{2}} K_{v}(2 \sqrt{\varphi \rho}) .
$$

By expanding Eq. (14) using Eq. (15), the PDF of $X_{t}$ takes the following final form:

$$
f_{X_{t}}\left(x_{t}\right)=\frac{2}{x_{t}}\left(\frac{\pi \phi x_{t}}{\mu_{t}}\right)^{\frac{\pi+\phi}{2}} \frac{1}{\Gamma(\pi) \Gamma(\phi)} K_{\phi-\pi}\left(2 \sqrt{\frac{\pi \phi x_{t}}{\mu_{t}}}\right),
$$

where $K_{v}(\cdot)$ is a modified Bessel function of the second kind of order $v$.

Eq. (16) is known by different names in the literature: Gamma-Gamma distribution (Al-Habash et al., 2001; Chatzidiamantis and Karagiannidis, 2009); generalized- $K$ distribution (Al-Ahmadi and Yanikomeroglu, 2010); generalized McDaniel model (Gu and Abraham, 2001); $K^{\prime}$ distribution (Teich and Diament, 1989); and so on. It is sometimes described as $K$-distribution (Blacknell, 1994; Redding, 
1999); however, the $K$-distribution is more widely known as a special case of Eq. (16) where $\pi=1$ (Abraham, 2003; Jakeman and Pusey, 1976). In this paper, we will use the term Gamma-Gamma distribution to refer to the PDF in Eq. (16). The Gamma-Gamma distribution is used in a number of areas for a statistical description of diverse scattering phenomena. Examples include: target and clutter scattering in radar (Lewinski, 1983); irradiance fluctuations in optics (Al-Habash et al., 2001; Teich and Diament, 1989); reverberation in sonar systems (Gu and Abraham, 2001); and fading and shadowing in wireless systems (Shankar, 2004).

\subsection{Properties of Gamma-Gamma Distribution}

The Gamma-Gamma distributed travel delay, denoted by $X_{t} \sim G G\left(\pi, \phi, \mu_{t}\right)$, is specified by three parameters: shape parameters $\pi$ and $\phi$ and mean $\mu_{t}$. For time and day boundary $(t=1, \cdots, T) \times(n=1, \cdots, N)$ within which the constant $C V$ assumption is acceptable, relations $C V\left[X_{t, n}\right]=1 / \sqrt{\pi}=\alpha$ and $C V\left[Y_{t}\right]=1 / \sqrt{\phi}=\beta$ hold and, thus, the two shape parameters characterize vehicle-to-vehicle variability and day-to-day variability through $\pi=1 / \alpha^{2}$ and $\phi=1 / \beta^{2}$, respectively $-\alpha$ and $\beta$ represent how quickly the SD of travel delay increases for every unit increase in its mean. Under this condition, $\pi$ and $\phi$ are not dependent on time interval $t$; the only time-dependent component is $\mu_{t}$, which accounts for time-of-day characteristics of travel delay. As such, once the model assumptions hold and we know $\pi$ and $\phi$, the entire PDF of travel delay at different time intervals can be obtained by measuring only the associated mean levels $\mu_{1}, \cdots, \mu_{T}$.

The $m^{\text {th }}$ moment of the Gamma-Gamma distribution is given by (Al-Ahmadi and Yanikomeroglu, 2010):

$$
E\left[X_{t}^{m}\right]=\frac{\mu_{t}^{m} \Gamma(\pi+m) \Gamma(\phi+m)}{\pi^{m} \phi^{m} \Gamma(\pi) \Gamma(\phi)} .
$$

Thus, the mean is simply $E\left[X_{t}\right]=\mu_{t}$ and the variance becomes:

$$
\operatorname{Var}\left[X_{t}\right]=E\left[X_{t}^{2}\right]-E\left[X_{t}\right]^{2}=\mu_{t}^{2} \frac{\pi+\phi+1}{\pi \phi}=\mu_{t}^{2}\left(\alpha^{2}+\beta^{2}+\alpha^{2} \beta^{2}\right)
$$

It is found that Eq. (18) coincides with the variance derived from the variance decomposition formula:

$$
\operatorname{Var}\left[X_{t}\right]=E_{n}\left[\operatorname{Var}_{i}\left[X_{t}\right]\right]+\operatorname{Var}_{n}\left[E_{i}\left[X_{t}\right]\right]
$$

where $E_{i}[\cdot]$ and $\operatorname{Var}_{i}[\cdot]$ stand for the expectation and SD across vehicles $\left(i=1, \cdots, v_{t, n}\right)$; and $E_{n}[\cdot]$ and $\operatorname{Var}_{n}[\cdot]$ stand for the expectation and SD across days $(n=1, \cdots, N)$. Eq. (19) indicates that the total variance is the sum of the day-to-day mean of vehicle-to-vehicle variances and the day-to-day variance of vehicle-to-vehicle means. Substituting $\operatorname{Var}_{i}[\cdot]=\alpha^{2} E_{i}[\cdot]^{2}$ and $\operatorname{Var}_{n}[\cdot]=\beta^{2} E_{n}[\cdot]^{2}$ from Eqs. (4) and (7) to Eq. (19) leads to $\operatorname{Var}\left[X_{t}\right]=\mu_{t}^{2}\left(\alpha^{2}+\beta^{2}+\alpha^{2} \beta^{2}\right)$ shown in Eq. (18).

\subsection{Gamma-Gamma Distribution with Time-varying Shape Parameters}


In the previous sections, we assumed that vehicle-to-vehicle and day-to-day CVs of travel delay ( $\alpha$ and $\beta$ ) are constant within given time and day boundary $(t=1, \cdots, T) \times(n=1, \cdots, N)$ and, thus, its shape parameters $\pi=1 / \alpha^{2}$ and $\phi=1 / \beta^{2}$ do not depend on $t$. This assumption, however, is restrictive as it is likely that there exist deviations from the linear proportional models in Eqs. (4) and (7) in real observations. This is especially true when the day-to-day mean-SD relationship exhibits a hysteresis loop, which leads the CV of travel delay (slope of the data points in mean-SD plot) to fluctuate around the theoretical trend line from period to period. In such cases, a more realistic Gamma-Gamma model could be derived by introducing two $\mathrm{CV}$ correction factors $\lambda_{t}^{\alpha}$ and $\lambda_{t}^{\beta}$, which are defined as the ratio of actual $\mathrm{CV}$ to theoretical CV such that

$$
\lambda_{t}^{\alpha}=\frac{\frac{1}{N} \sum_{n=1}^{N} C V\left[X_{t, n}\right]}{\alpha}
$$

and

$$
\lambda_{t}^{\beta}=\frac{C V\left[Y_{t}\right]}{\beta},
$$

respectively.

Multiplying static $\alpha$ and $\beta$ by the associated correction factors leads to $\lambda_{t}^{\alpha} \alpha$ and $\lambda_{t}^{\beta} \beta$, which capture the time-dependent CVs more correctly. These will then allow the shape parameters to be adjusted accordingly through $\pi_{t}=1 /\left(\lambda_{t}^{\alpha} \alpha\right)^{2}$ and $\phi_{t}=1 /\left(\lambda_{t}^{\beta} \beta\right)^{2}$, respectively.

An example is provided in FIGURE 3 to illustrate how $\beta$-correction factor $\lambda_{t}^{\beta}$ could be used to capture a hysteresis loop in day-to-day mean-SD relationship in modeling travel time using the GammaGamma distribution. FIGURE 3 (a) depicts a counter-clockwise loop on the day-to-day mean-SD plot, where the slope (i.e., $\mathrm{CV}$ ) at $t$ (during congestion onset) is lower than the overall trend $\beta$ (i.e., $\lambda_{t}^{\beta} \beta<\beta$ ), while the slope at $t+k$ (during congestion offset) is higher than the overall trend $\beta$ (i.e., $\lambda_{t+k}^{\beta} \beta>\beta$ ). If we plot the value of $\lambda_{t}^{\beta}$ with respect to $t$, we would obtain its temporal profile similar to the graph in FIGURE 3 (b). If we could identify a theoretical functional form or empirical trend that allows us to describe $\lambda_{t}^{\beta}$ as a function of $t$, then we would be able to apply this extended Gamma-Gamma model to capture complex time-of-day effects in the statistical representation of travel time distribution, without adding too much complexity to the model specification. This will also allow us to investigate how hysteresis patterns affect the resulting travel time distribution in a more systematic manner. 


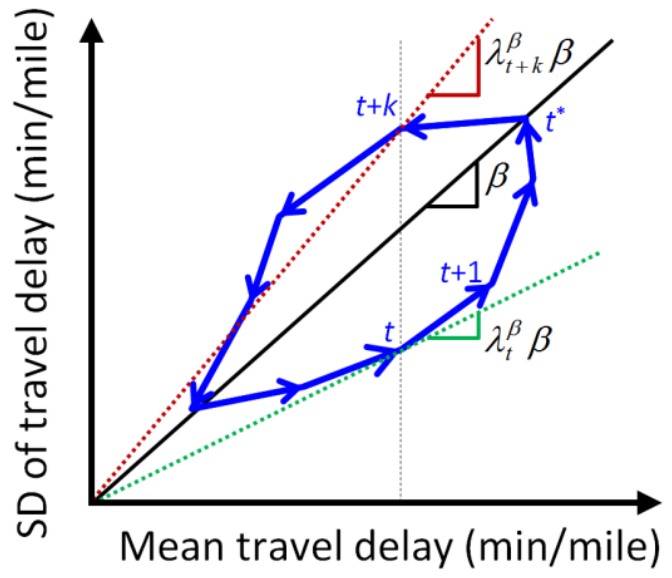

(a)

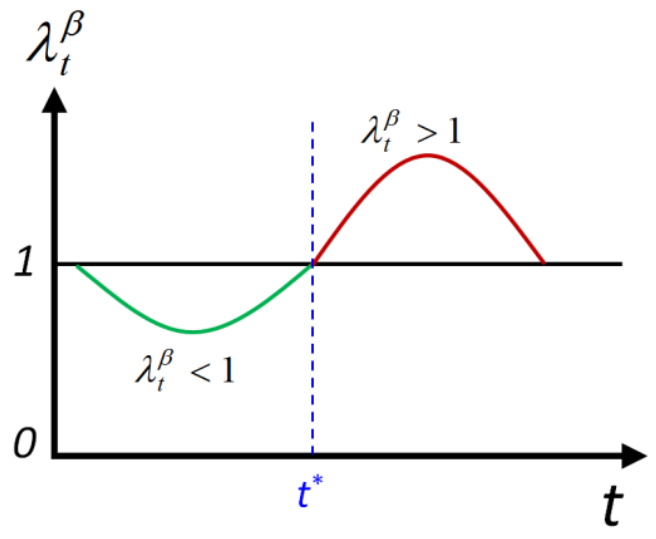

(b)

FIGURE 3 Illustrative example of (a) hysteresis loop in day-to-day mean-SD relationship in travel delay and (b) the associated time-varying slope (or $\mathrm{CV}$ ) profile; day-to-day $\mathrm{CV}$ correction factor $\lambda_{t}^{\beta}$ can be used to capture time-dependent deviations from global slope $\beta$.

\section{ANALYSIS OF SIMULATED TRAJECTORIES}

\subsection{Data}

A simulation experiment was conducted to demonstrate the procedures for calibrating the GammaGamma model and its application in modeling travel time distribution across the entire vehicle population over a long-term evaluation period. The simulation was performed using a particle-based dynamic traffic simulation-assignment model (Mahmassani and Sbayti, 2009), with time-varying OD demand calibrated by using historical data. FIGURE 4 presents the simulation network used for this study, which covers roadways in Chicago, Illinois, USA consisting of 1,578 nodes, 4,805 links, and 218 zones. Daily scenarios were prepared for morning peak from 6AM to 10AM using historical weather and accident data in 2010. The weather data were collected from the Automated Surface Observing System (ASOS) station at Chicago Midway International Airport and the accident data were obtained from the Illinois Department of Transportation. A total of 261 daily scenarios $(N=261)$ representing workdays in 2010 were created and simulated. Each scenario produces approximately 521,000 vehicle trajectories during the 4-hour simulation horizon. Additional information on the network development and calibration process is available in (Mahmassani et al., 2012b; Verbas et al., 2011).

The analysis is performed at two different aggregation levels, namely, network and OD. At the network-level, we divide the entire simulation horizon into a series of 5 -minute intervals $(T=48)$. For each scenario, vehicles are divided into 48 groups based on their departure times, producing a total of $48 \times 261=12,528$ vehicle groups. The number of vehicles in each group ranges between 7,472 and 14,568. To perform the OD-level analysis, we select one OD-pair with the average distance of 10.8 miles, depicted as $\mathrm{O} \rightarrow \mathrm{D}$ in FIGURE 4. A 15 -minute time interval is used ( $T=16)$ to ensure that each timeinterval group contains a sufficient number of observations. For each simulation run, vehicles belonging to the selected OD are divided into these 16 groups based on their departure times yielding a total of $16 \times 261=4,176$ vehicle groups. The number of vehicles in each group ranges between 25 and 110 . 


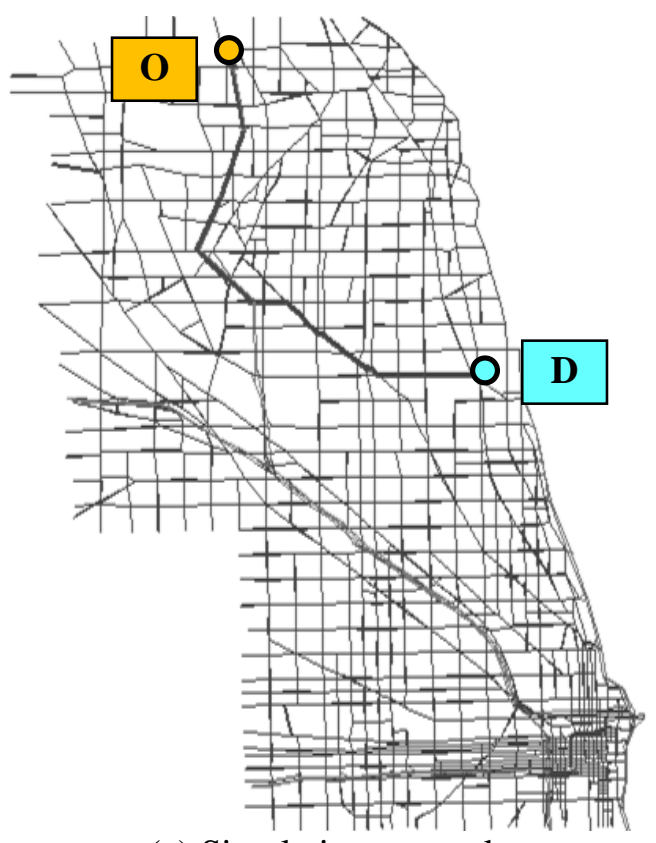

(a) Simulation network

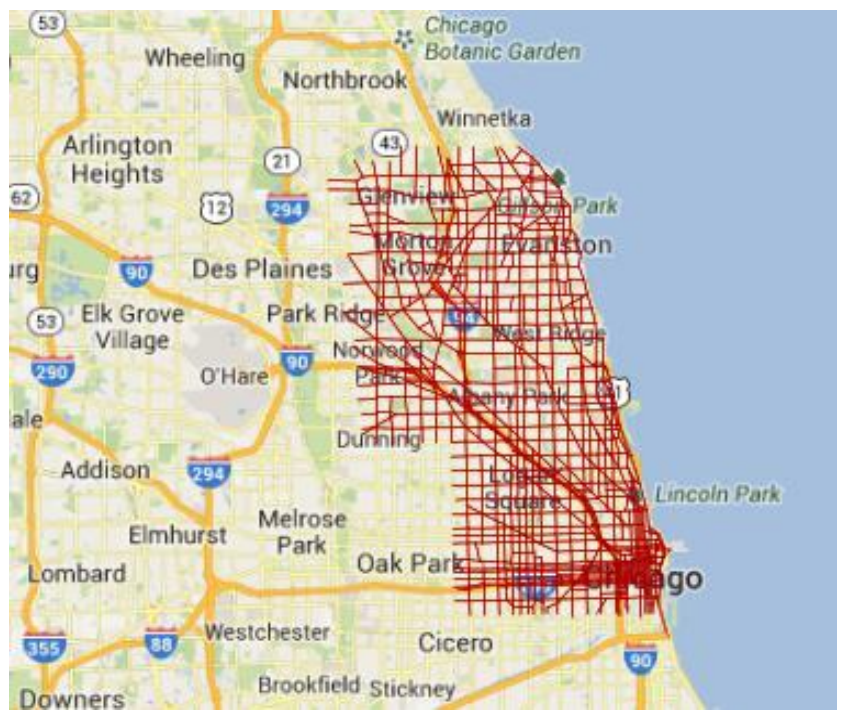

(b) Google Map

FIGURE 4 Chicago network and selected OD-pair for simulation study.

\subsection{Estimating Parameters of Linear Model for Mean Travel Delay and Its Standard Deviation}

Using the simulated trajectory data, we estimate the parameters of the linear (proportional) model for the mean and SD of travel delay in Eqs. (4) and (7) as follows:

1. For $t=1, \cdots, T$ and $n=1, \cdots, N$, calculate the mean and SD of travel time and produce one sample point per each $(t, n)$ cell.

2. Given a set of $T \times N$ sample points, estimate the minimum travel time per unit distance $\left(-\theta_{1} / \theta_{2}\right.$ in Eq. (1) ) by performing linear regression.

3. Obtain travel delay $x_{t, n, i}$ by subtracting the minimum travel time from each travel time observation.

4. For $t=1, \cdots, T$ and $n=1, \cdots, N$, calculate the vehicle-to-vehicle mean $\left(\hat{\mu}_{X_{t, n}}\right)$ and $\operatorname{SD}\left(\hat{\sigma}_{X_{t, n}}\right)$ of travel delay using Eqs. (2) and (3), respectively.

5. Estimate $\alpha$ in Eq. (4) based on $T \times N$ pairs of $\left(\hat{\mu}_{X_{t, n}}, \hat{\sigma}_{X_{t, n}}\right)$ from Step 4 by fitting a line that passes through the origin.

6. For $t=1, \cdots, T$, calculate the day-to-day mean $\left(\hat{\mu}_{t}\right)$ and $\operatorname{SD}\left(\hat{\sigma}_{t}\right)$ using Eqs. (5) and (6), respectively.

7. Estimate $\beta$ in Eq. (7) based on $T$ pairs of $\left(\hat{\mu}_{t}, \hat{\sigma}_{t}\right)$ from Step 6 by fitting a line that passes through the origin.

The estimation results for both network- and OD-levels are presented in TABLE 2. The estimated values for the minimum travel time per unit distance are $1.27 \mathrm{~min} / \mathrm{mile}$ at the network level and $1.36 \mathrm{~min} / \mathrm{mile}$ at the OD-level. TABLE 2 shows the linear regression results, which include the least-squares (LS) estimates of slope coefficients $\alpha$ and $\beta$, their standard errors, and the coefficient of determination $\left(R^{2}\right)$ values. It is noted that the definition of $R^{2}$ for a regression model without an intercept is controversial and 
different formula may be used depending on authors (Eisenhauer, 2003). This paper uses the general definition $R^{2} \equiv 1-S S_{\text {err }} / S S_{\text {tot }}=1-\sum_{\mathrm{i}}\left(\mathrm{y}_{\mathrm{i}}-\hat{\theta} \mathrm{x}_{\mathrm{i}}\right)^{2} / \sum_{\mathrm{i}}\left(\mathrm{y}_{\mathrm{i}}-\overline{\mathrm{y}}\right)^{2}$, given a linear model with no intercept in the form $\mathrm{y}_{\mathrm{i}}=\theta \mathrm{x}_{\mathrm{i}}+\varepsilon_{\mathrm{i}}$, rather than an alternative definition $R^{2} \equiv \sum_{\mathrm{i}}\left(\hat{\theta} \mathrm{x}_{\mathrm{i}}\right)^{2} / \sum_{\mathrm{i}}\left(\mathrm{y}_{\mathrm{i}}\right)^{2}$ used in some statistical software packages. The $R^{2}$ values derived here can serve as the usual diagnostic measure. The results in TABLE 2 show that the linear proportional model has high goodness-of-fit in all four cases, producing high $R^{2}$ values. FIGURE 5 provides mean-SD plots along with the fitted lines. The overall results suggest that the relationship between mean and SD of travel delay is well characterized by a simple proportional model in both vehicle-to-vehicle and day-to-day distributions, though some decree of heteroscedasticity exists in the vehicle-to-vehicle cases. For both network- and OD-levels, $\hat{\alpha}$ is greater than $\hat{\beta}$, indicating that the vehicle-to-vehicle $\mathrm{CV}$ is greater than the day-to-day $\mathrm{CV}$. When comparing the relative magnitude between $\hat{\alpha}$ and $\hat{\beta}$ through $\hat{\beta} / \hat{\alpha}$, we observe that the ratio is much smaller at the network-level ( $\hat{\beta} / \hat{\alpha}=0.07$ ) compared to the OD-level ( $\hat{\beta} / \hat{\alpha}=0.27$ ), primarily due to the relatively small value of $\hat{\beta}$ at the network-level $(\hat{\beta}=0.057)$. The small value of $\hat{\beta}$ at the network-level could be explained as the result of obtaining $Y_{t}$ by taking the average of $X_{t, n}$ over a large spatial boundary, which tend to smoothe out facility-specific day-to-day fluctuations and lead to a small degree of network-level day-to-day fluctuations. This provides an implication that the relative contribution of day-to-day variability to the total variance, captured by Eq. (18), depends on the network scale (e.g., decreases as the network scale increases) and the selection of the network scale is important in assessing the sources of travel time variability. Further research should be conducted, however, to fully understand the effects of network scale on vehicle-to-vehicle and day-to-day variability characteristics.

TABLE 2 Parameter Estimation Results based on Simulated Vehicle Trajectory Data for the Chicago Network at the Network-level and OD-level

\begin{tabular}{|c|c|c|c|c|}
\hline \multicolumn{3}{|c|}{ Parameter Estimation } & Network-level & OD-level \\
\hline \multicolumn{3}{|c|}{$\begin{array}{c}\text { Minimum Travel Time } \\
\text { per Unit Distance (min/mile) }\end{array}$} & 1.27 & 1.36 \\
\hline \multirow{6}{*}{$\begin{array}{c}\text { Linear } \\
\text { Model } \\
\text { Parameters }\end{array}$} & \multirow{3}{*}{ veh-to-veh } & $\hat{\alpha}$ (LS estimate) & 0.832 & 0.970 \\
\hline & & std. error & 0.001 & 0.005 \\
\hline & & $R^{2}$ & 0.874 & 0.703 \\
\hline & \multirow{3}{*}{ day-to-day } & $\hat{\beta}$ (LS estimate) & 0.057 & 0.263 \\
\hline & & std. error & 0.002 & 0.016 \\
\hline & & $R^{2}$ & 0.669 & 0.651 \\
\hline \multirow{6}{*}{$\begin{array}{c}\text { Gamma } \\
\text { Distribution } \\
\text { Parameters }\end{array}$} & & $\hat{\pi}_{M L}$ (ML estimate) & 1.521 & 1.426 \\
\hline & veh-to-veh & $\hat{\alpha}_{M L}\left(=1 / \sqrt{\hat{\pi}_{M L}}\right)$ & 0.811 & 0.837 \\
\hline & \multirow{4}{*}{ day-to-day } & $\hat{\alpha}_{M L} / \hat{\alpha}$ & 0.974 & 0.864 \\
\hline & & $\hat{\phi}_{M L}($ ML estimate $)$ & 206.4 & 8.955 \\
\hline & & $\hat{\beta}_{M L}\left(=1 / \sqrt{\hat{\phi}_{M L}}\right)$ & 0.070 & 0.334 \\
\hline & & $\hat{\beta}_{M L} / \hat{\beta}$ & 1.213 & 1.270 \\
\hline
\end{tabular}


Network-level

(a)

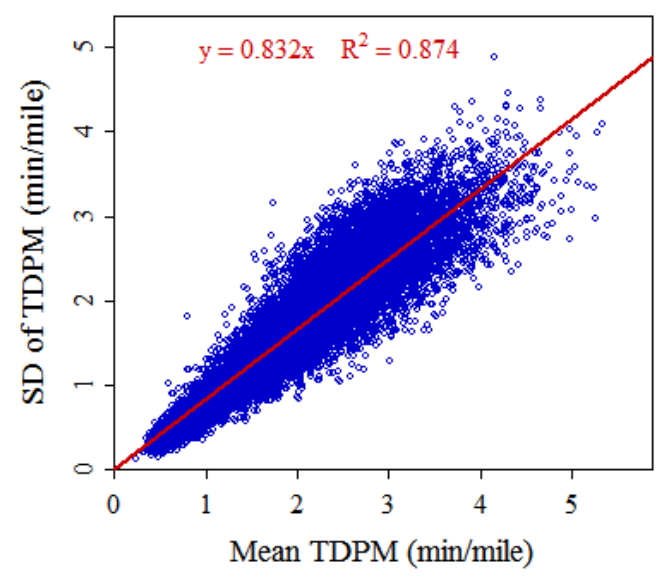

(c)

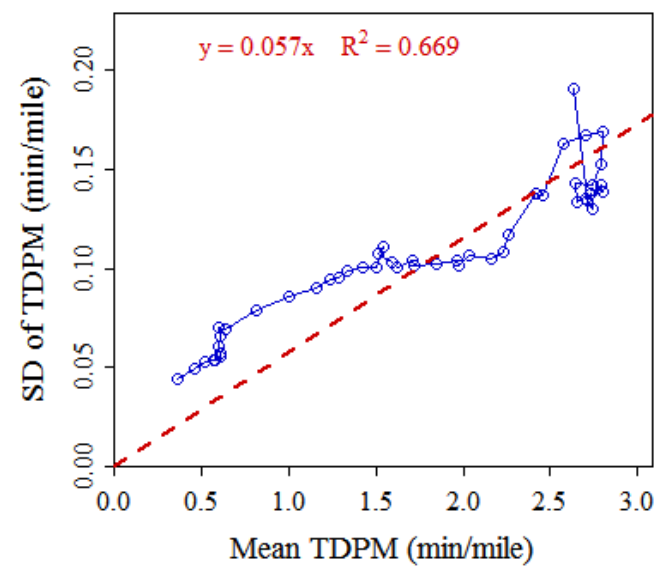

(e)

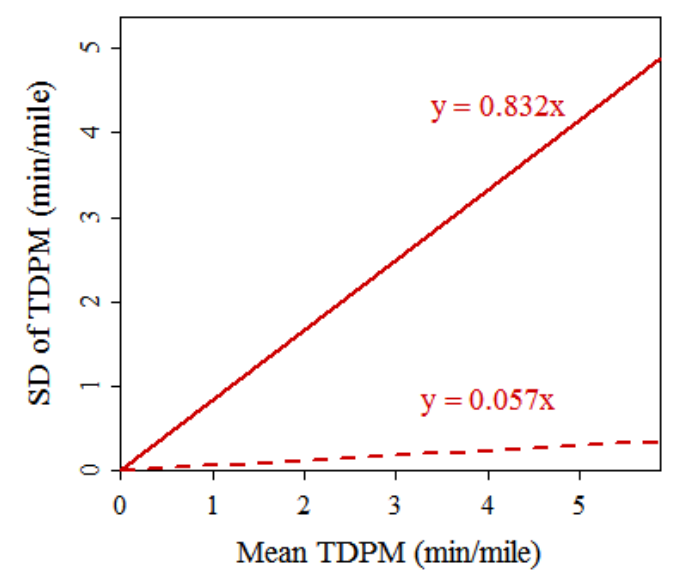

(b)

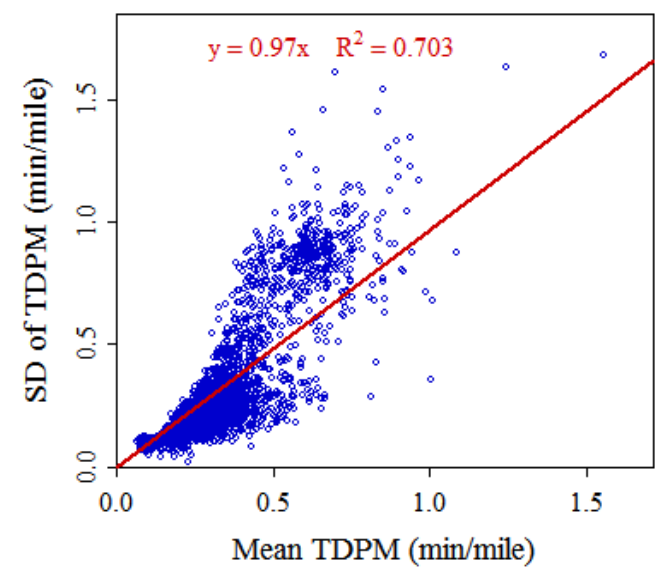

(d)

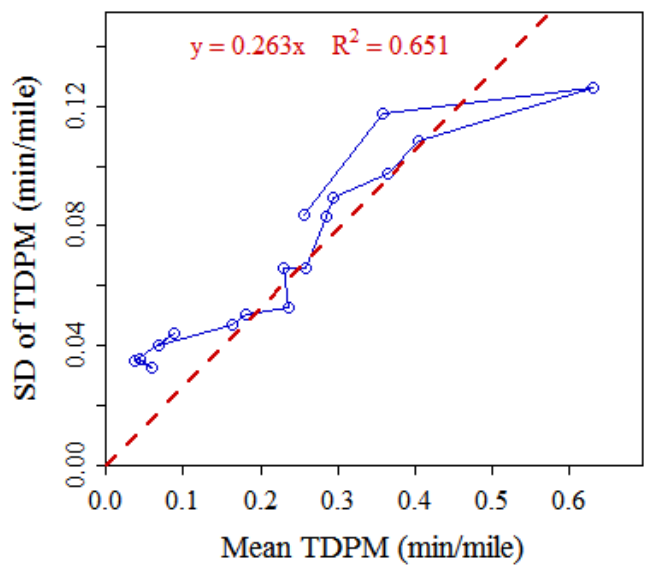

(f)

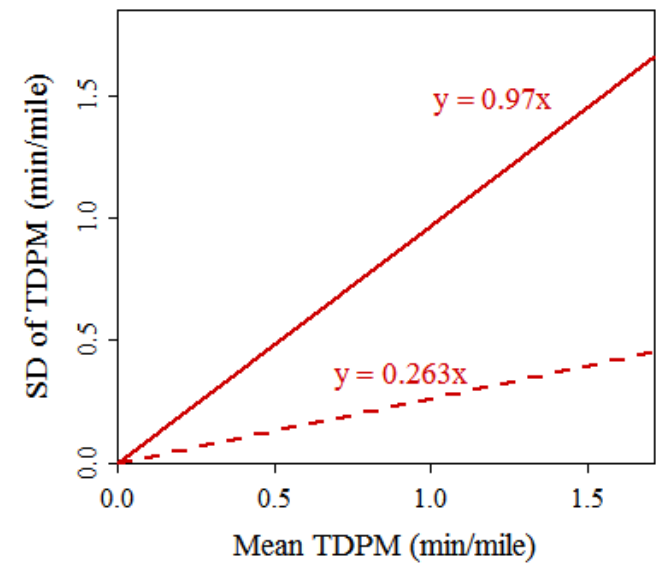

FIGURE 5 Relation between mean and SD of travel delay per mile (TDPM) in vehicle-to-vehicle and day-to-day distributions, constructed at network-level (left) and OD-level (right) using simulated data from the Chicago network: (a,b) vehicle-to-vehicle; (c,d) day-to-day; and (e,f) both. 


\subsection{Estimating Parameters of Component Gamma Distributions}

Given the estimated travel delay measures, we can estimate $\pi$ and $\phi$ from the associated component Gamma distributions, i.e., $\gamma \sim \operatorname{Gamma}(\pi, 1 / \pi)$ and $\eta \sim \operatorname{Gamma}(\phi, 1 / \phi)$, respectively. At each level (network and OD), we perform the following procedure to obtain the error samples $(\hat{\gamma}$ and $\hat{\eta})$ and the associated Gamma distribution parameters $(\hat{\pi}$ and $\hat{\phi})$ :

1. For $t=1, \cdots, T$, calculate $\hat{\mu}_{t}$ using Eq. (5).

2. For $t=1, \cdots, T, n=1, \cdots, N$, and $i=1, \cdots, v_{t, n}$, calculate the error of individual travel delay, $\hat{\gamma}$, using Eq. (8).

3. Fit a Gamma distribution to a total of $\sum_{n=1}^{N} \sum_{t=1}^{T} v_{t, n}$ points of $\hat{\gamma}$ and obtain the maximum likelihood (ML) estimate of shape parameter $\pi$.

4. For $t=1, \cdots, T$ and $n=1, \cdots, N$, calculate the error of mean travel delay $\hat{\eta}$ using Eq. (9).

5. Fit a Gamma distribution to $T \times N$ points of $\hat{\eta}$ and obtain the ML estimate of shape parameter $\phi$.

The ML estimates of the Gamma shape parameters obtained above, denoted by $\hat{\pi}_{M L}$ and $\hat{\phi}_{M L}$, are presented TABLE 2 to check if theoretical relations $C V\left[X_{t, n}\right]=1 / \sqrt{\pi}=\alpha$ and $C V\left[Y_{t}\right]=1 / \sqrt{\phi}=\beta$ hold. It is noted that $\hat{\pi}_{M L}$ and $\hat{\phi}_{M L}$ were estimated using the error data combined from all time intervals and days and represent the static shape parameters for the basic Gamma-Gamma model. By denoting the CV estimates of the Gamma distributions by $\hat{\alpha}_{M L}=1 / \sqrt{\hat{\pi}_{M L}}$ and $\hat{\beta}_{M L}=1 / \sqrt{\hat{\phi}_{M L}}$, respectively, we compute ratios $\hat{\alpha}_{M L} / \hat{\alpha}$ and $\hat{\beta}_{M L} / \hat{\beta}$ for a comparison. The results presented in TABLE 2 show that the values are close to 1 , where $\hat{\alpha}_{M L} / \hat{\alpha}$ for the network- and OD-levels are 0.974 and 0.864 ; and the associated $\hat{\beta}_{M L} / \hat{\beta}$ are 1.213 and 1.270 , respectively, though some degree of deviation exists. A source of such deviations may come from the estimation of the minimum travel time per unit distance (i.e., $-\theta_{1} / \theta_{2}$ ), which is obtained by finding a common $\mathrm{x}$-intercept for both vehicle-to-vehicle and day-to-day mean-SD trend lines. A more sophisticated method could be applied to find the optimal minimum travel time that maximizes the overall goodness-of-fit of the final proportional models. This will be addressed as part of future research.

This paper presents the estimation procedure that estimates the shape parameters from each component separately. One could instead estimate them jointly from the compound PDF in Eq. (16). It is, however, noted that the ML estimate for the parameters of the Gamma-Gamma distribution has no closed form and, thus, alternative approaches such as method of moments (MOM) (Wang and Cheng, 2010) and numerical approximation (Kazeminia and Mehrjoo, 2013) are required. Exploring joint estimators for the parameters of the Gamma-Gamma distribution is beyond the scope of this study and will be conducted in our future work.

\subsection{Evaluating Performance of Gamma-Gamma Distribution}

Given the estimates of the two shape parameters $\left(\hat{\pi}=1 / \hat{\alpha}^{2}\right.$ and $\left.\hat{\phi}=1 / \hat{\beta}^{2}\right)$ and the mean parameters for $T$ time intervals $\left(\hat{\mu}_{1}, \cdots, \hat{\mu}_{T}\right)$, we can construct the overall distribution of travel delay per unit distance $X_{t}$ - both across vehicles and across days - for all $T$ time intervals using the Gamma-Gamma PDF in Eq. 
(16). In this section, we examine the performance of the Gamma-Gamma distribution using OD-level data. The performance of the model is compared with other two commonly used models, namely, Gamma and Lognormal distributions. For the Gamma and Lognormal distributions, the parameters are estimated using the travel delay data from all $T$ time intervals to find the best-fit distributions for the $(t=1, \cdots, T) \times(n=1, \cdots, N)$ boundary. Since the number of parameters differs among the tested distributions, the relative goodness-of-fit of each model is measured using the Akaike Information Criterion (AIC) defined as $2 k-2 \ln (L)$, where $k$ is the number of parameters and $L$ is the maximized value of the likelihood function for the estimated model. For each model, the AIC is calculated based on the log-likelihoods from the entire time span to assess the overall goodness-of-fit for $(t=1, \cdots, T) \times(n=1, \cdots, N)$. The AIC of each model is then subtracted by the AIC of the best candidate model to obtain the AIC difference, i.e., $\triangle A I C_{j}=A I C_{j}-\min (A I C)$ to express the relative performance of model $j$. The smaller $\triangle A I C_{j}$ is, the better model $j$ describes the data; the model estimated to be best has $\triangle A I C_{j}=0$. In addition to the overall goodness-of-fit measure, the relative log-likelihood values are calculated at each $t$ to examine the models' relative performance at individual time intervals. The relative log-likelihood is defined as $L L_{j}-\overline{L L}$, where $L L_{j}$ represents the log-likelihood under model $j$ and $\overline{L L}$ represents the mean of the log-likelihood values from all three models. The greater the value of $L L_{j}-\overline{L L}$ is, the better model $j$ fits the data at a given time interval. Since the sample size varies from period to period, the log-likelihood values cannot be directly compared across time intervals; we can only examine the relative rankings within each time interval. FIGURE 6 presents the values of $\triangle A I C_{j}$ and $L L_{j}-\overline{L L}$ for all three models. The results show that the Gamma-Gamma distribution has the best overall goodnessof-fit performance $\left(\triangle A I C_{j}=0\right)$ despite the penalty due to the extra parameters - the number of parameters in the Gamma-Gamma distribution is $k=2+T=18$, while $k=2$ for the Gamma and Lognormal distributions. The figure also shows that the log-likelihood under the Gamma-Gamma distribution is consistently higher than that of the Gamma or Lognormal distribution throughout the time span. The Gamma distribution shows a similar performance around 7:15 and 8:15, but the performance drops at the beginning and at the end. The Lognormal distribution shows a relatively poor fit for the overall period. FIGURE 7 provides detailed histograms of travel delay with the fitted density curves for selected time intervals: (a) 7:00, (b) 8:00, and (c) 9:00. We observe that, given the two shape parameters ( $\pi$ and $\phi$ ) characterizing the vehicleto-vehicle and day-to-day variability of the overall time span, the Gamma-Gamma distribution is capable of adapting to the temporal evolution of the travel delay distribution through the time-varying mean parameters $\left(\mu_{1}, \cdots, \mu_{T}\right)$. While flexibility often comes at the cost of increased complexity, the GammaGamma model offers this without adding much complexity as it only requires the estimation of additional mean parameters, which are relatively easy to measure or predict. This level of flexibility is not provided by the traditional probability distributions, which would fail to describe the travel time distribution for certain time intervals if the travel time distribution varies largely during the period where a model was calibrated. 


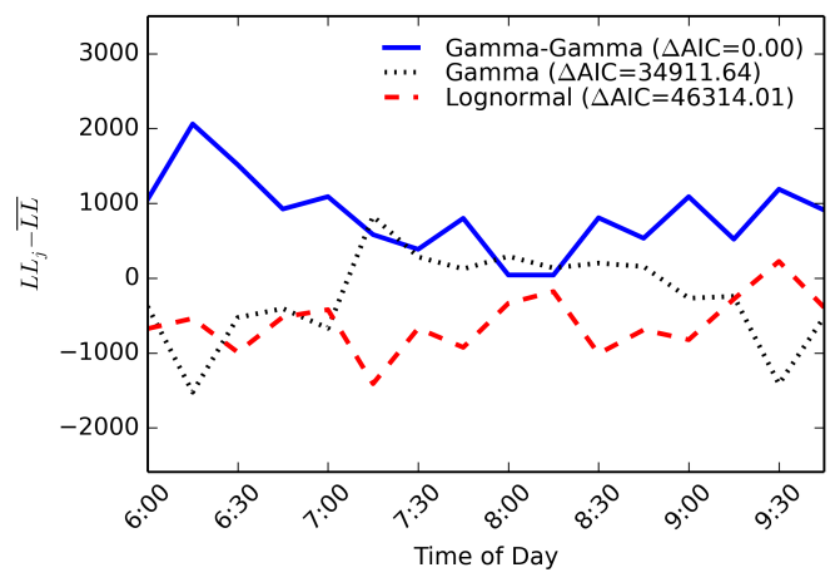

FIGURE 6 Relative values of log-likelihood under Gamma-Gamma, Gamma, and Lognormal distributions fitted to simulated vehicle trajectory data.

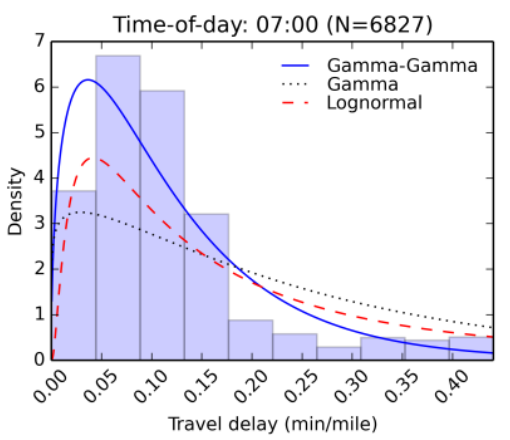

(a)

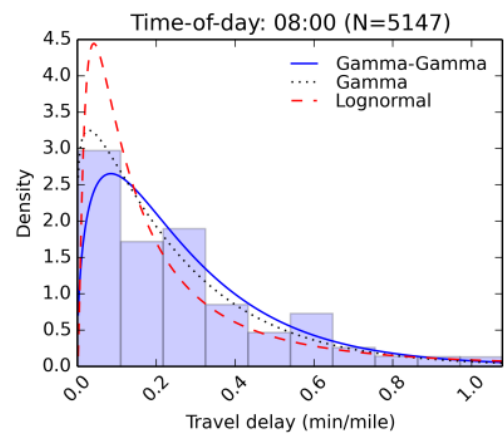

(b)

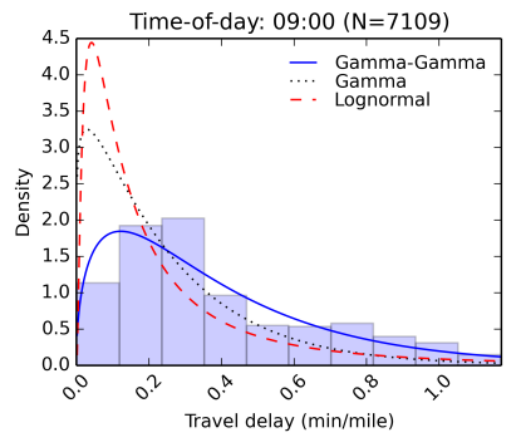

(c)

FIGURE 7 Travel delay distribution (across vehicles and days) and the fitted density curves; data are from the simulated vehicle trajectories at OD-level for time intervals: (a) 7:00, (b) 8:00, and (c) 9:00.

\section{VALIDATION USING VEHICLE PROBE DATA}

\subsection{Data}

Validation of the proposed model requires real-world individual trip data with a sufficient number of observations within each time interval as well as across days. The availability of such data is limited especially for the same study area as the simulation. Alternatively, data from other networks can be used to validate the model correctness. This paper uses data from the Strategic Highway Research Program 2 (SHRP2) project L04 (Mahmassani et al., 2013b). The dataset contains individual vehicles' trajectories collected from TomTom Global Positioning System (GPS) devices during a two-week period in May 2010 (May 2 - May 17) in New York, NY, USA. For this study, we select three target networks for which travel time data are extracted and the mean-SD relationships are analyzed. FIGURE 8 shows these selected networks. Network $A$ covers the Manhattan area, which consists of grid-like dense city streets, 
two highways along the east and west sides of Manhattan, and two underwater tunnels (Lincoln Tunnel and Queens-Midtown Tunnel). Network $B$ covers the Brooklyn area that has mainly arterial roads and residential streets, without significantly encompassing major highways. Finally, Network $C$ contains the area bounded by four major highways, where two (Interstates 678 and 295) run north-south and the other two (Interstate 495 and Grand Central Parkway) run east-west. The network boundary of all three areas is roughly 3.7 miles $\times 3.7$ miles $(6 \mathrm{~km} \times 6 \mathrm{~km})$. For each network, we identify vehicle trajectories that pass the given area and extract the portion of travel times spent traveling within the network boundary. Travel time data with travel distance shorter than 0.62 miles $(1 \mathrm{~km})$ were excluded from the analysis as those might be too short to capture the usual travel time characteristics in the given networks. The time period of analysis considered in this study is a PM period between 1 PM and $8 \mathrm{PM}$, which contains the entire cycle of network loading and unloading phases. Each day was divided into 30-minute intervals - hence a total of 14 intervals $(T=14)$ - and travel time data were grouped into these 14 time-interval groups based on vehicles' network entrance time (i.e., the time when each vehicle starts its trip within the network). This yields a total of 224 time-interval groups during 16 days $(N=16)$ for each network. TABLE 3 provides summary statistics for the data samples constructed for the three study networks. The average number of travel time observations at each time interval ranges from 26 to 57 . The average travel distance is similar in all three networks (i.e., 2.55-2.96 miles) but the average travel time decreases in the order of A $(13.2 \mathrm{~min})>\mathrm{B}(12.3 \mathrm{~min})>\mathrm{C}(6.7 \mathrm{~min})$. The sample mean of travel time per unit distance is estimated as $5.83 \mathrm{~min} / \mathrm{mile}, 5.33 \mathrm{~min} / \mathrm{mile}$, and $3.08 \mathrm{~min} / \mathrm{mile}$ for networks $\mathrm{A}, \mathrm{B}$, and $\mathrm{C}$, respectively, showing that the distance-normalized travel time in networks A and B are nearly twice that in network C. Roughly speaking, networks A and B in Manhattan and Brooklyn could be characterized as a low-speed urban street network, whereas network $\mathrm{C}$ in Queens can be viewed as a freeway network that offers relatively high free-flow speeds.

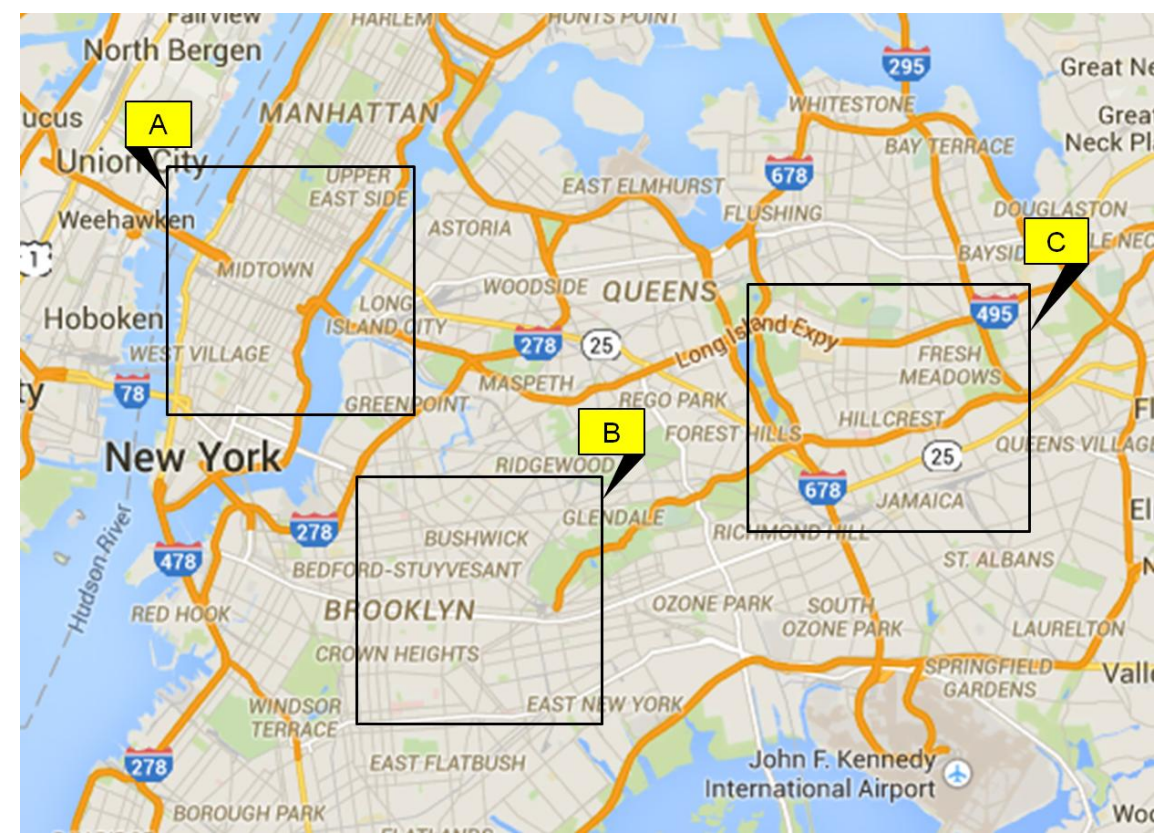

FIGURE 8 Selected study networks in New York, NY, USA: networks A, B, and C cover a $3.7 \mathrm{mi} \times 3.7 \mathrm{mi}(6 \mathrm{~km} \times 6 \mathrm{~km})$ area in Manhattan, Brooklyn, and Queens, respectively. 
TABLE 3 Description of Vehicle Probe Data Samples for Model Validation

\begin{tabular}{lccc}
\hline & Network & Network & Network \\
& A & B & C \\
\hline Total Number of Vehicle Trajectories & 12,264 & 5,758 & 12,860 \\
(between13:00 and 20:00 during 16 days) & 55 & 26 & 57 \\
Average Sample Size per Time Interval & 13.19 & 12.27 & 6.72 \\
Average Travel Time (min) & 2.96 & 2.55 & 2.85 \\
Average Travel Distance (miles) & 5.83 & 5.33 & 3.08 \\
Average Travel Time per Unit Distance (min/mile) & &
\end{tabular}

\subsection{Validating Model Assumptions}

Using the actual vehicle probe data described above, we validate two main assumptions made in deriving the Gamma-Gamma model: (i) the linear (proportional) relationship between mean and SD of travel delay in Eqs. (4) and (7) and (ii) the Gamma-distributed i.i.d. error assumptions in Eqs. (8) and (9).

For validating the linearity assumption, we estimate the minimum travel time per unit distance and slope parameters $\alpha$ and $\beta$ for each of the three networks, following the procedure described in Section 4.2. TABLE 4 provides the parameter estimation results. The minimum travel time for networks A, B, and $\mathrm{C}$ are estimated as $1.91 \mathrm{~min} / \mathrm{mile}, 1.62 \mathrm{~min} / \mathrm{mile}$, and $0.49 \mathrm{~min} / \mathrm{mile}$, respectively, which show a similar pattern as in Average Travel Time per Unit Distance in TABLE 3 (i.e., A > B > C). Given the obtained travel delay measures, FIGURE 9 presents mean-SD plots for both vehicle-to-vehicle and dayto-day distributions, with the fitted linear models. The results show that, in all three networks, the probe data exhibit a pattern that is consistent with the simulated trajectory data; that is, the SD of travel delay is highly positively correlated with its mean and the relationship between two is adequately characterized by a linear model. The $R^{2}$ values for $\hat{\alpha}$ range from 0.447 to 0.587 and those for $\hat{\beta}$ range from 0.361 to 0.404 . Network B has the mildest slope in both vehicle-to-vehicle and day-to-day mean-SD relations. The steepest slope in the vehicle-to-vehicle plot is found in network A and the steepest slop in the day-to-day plot is found in network $\mathrm{C}$.

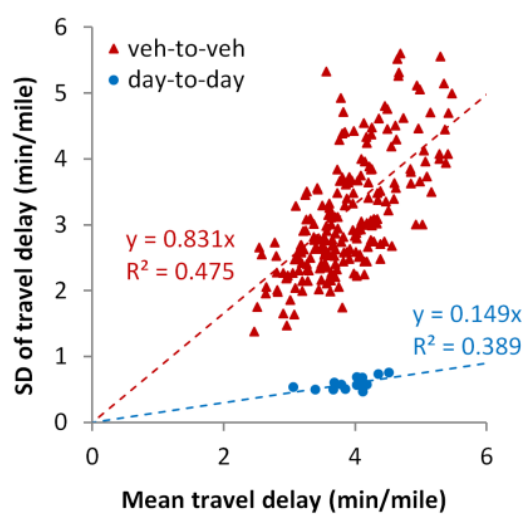

(a)

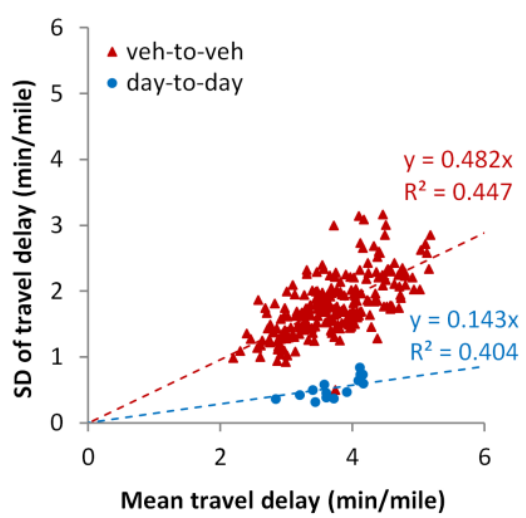

(b)

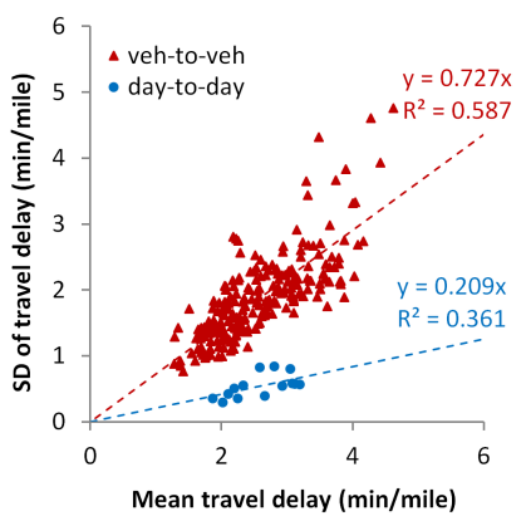

(c)

FIGURE 9 Relationship between mean and SD of travel delay per unit distance in vehicle-to-vehicle and day-to-day distributions, constructed using actual trajectory data from the selected three networks in the New York region: (a) network A, (b) network B, and (c) network $\mathrm{C}$. 
TABLE 4 Parameter Estimation Results based on Actual Vehicle Trajectory Data for Three Study Networks A, B, and $\mathrm{C}$ in the New York region

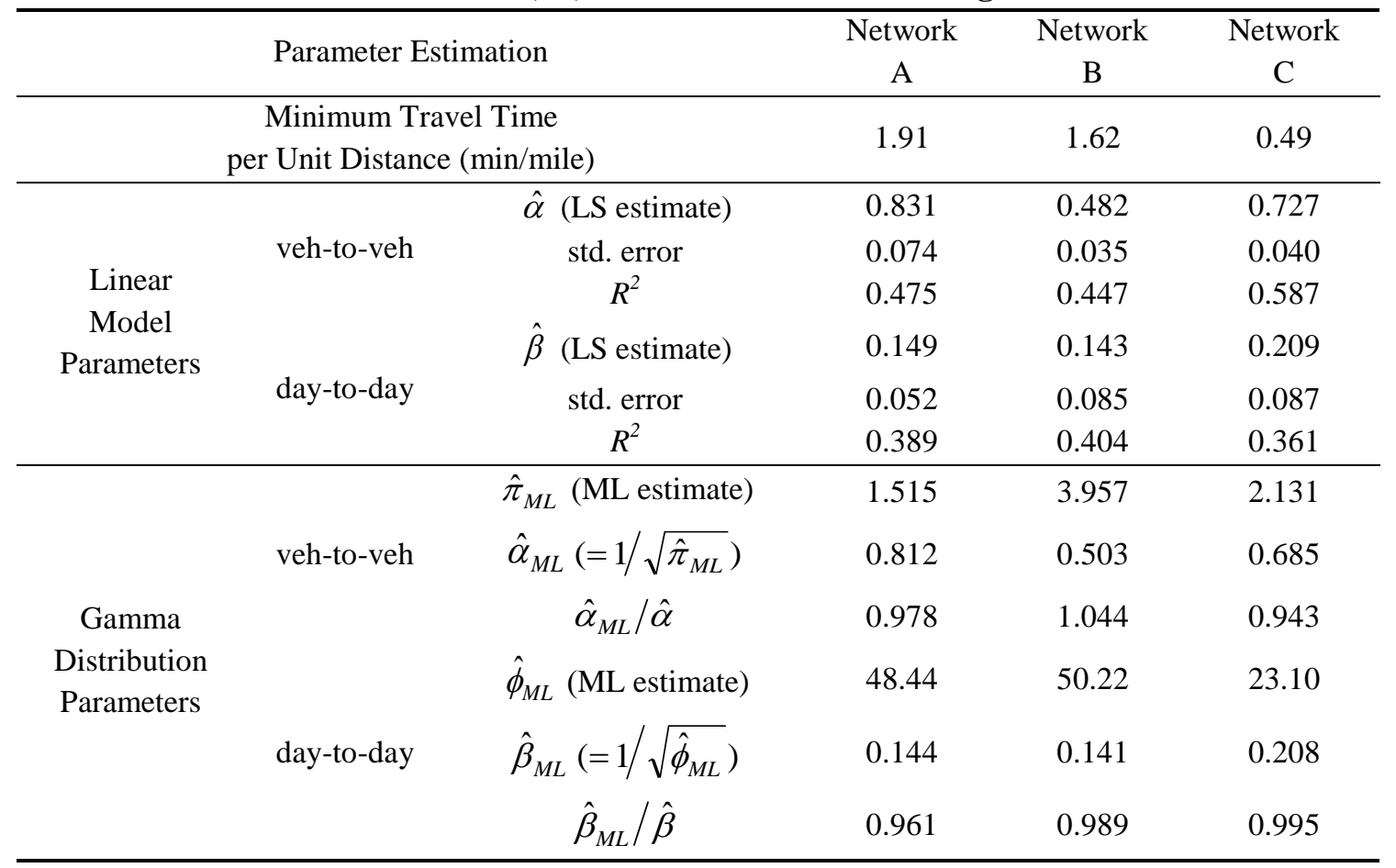

For validating the Gamma-distributed i.i.d. error assumption, we first obtain the error samples ( $\hat{\gamma}$ and $\hat{\eta}$ ) across all $t$ and $n$ by conducting the procedure described in Section 4.3. The error sample of each component is then tested against its associated best-fit Gamma distribution using the one-sample Kolmogorov-Smirnov (KS) test. TABLE 5 (a) presents the estimated shape parameters of the best-fit Gamma distributions, KS test statistics, and $p$-values, respectively. The test results in TABLE 5 (a) show that in all cases the null hypothesis $\left(\mathrm{H}_{0}\right)$ that the error term follows a Gamma distribution cannot be rejected at the significance level of $5 \%$ as the $p$-values are greater than 0.05 . In the case of day-to-day error, the null hypothesis cannot be rejected even at the stricter condition of $10 \%$ significance level. Next, we validate the assumption that the errors are i.i.d across $t$ and $n$. To achieve this, we split the vehicle-tovehicle error sample according to $t$ and $n$ and day-to-day error sample according to $t$ and test whether the distribution of error is the same across splits. First, we split the sample of $\hat{\gamma}$ into a total of 224 time-andday groups and $\hat{\eta}$ into a total of 14 time-of-day groups. Then, we conduct the one-sample KS test for each error group against its associated best-fit Gamma distribution, which was obtained based on the pooled samples above. TABLE 5 (b) presents the test results. For network A, in 219 out of 224 vehicleto-vehicle error groups (97.8\%), the null hypothesis that errors are from the same Gamma distribution $\operatorname{Gamma}(\pi, 1 / \pi)$ cannot be rejected at the significance level of 5\%. Similarly, for networks B and C, the null hypothesis cannot not be rejected in $100 \%$ and $82.6 \%$ of the tested vehicle-to-vehicle error groups, respectively. For the case of day-to-day error, all three networks show that the null hypothesis that errors are from the same Gamma distribution $\operatorname{Gamma}(\phi, 1 / \phi)$ cannot be rejected at $5 \%$ significance level in all 
14 time-of-day error groups. Based on the test results in TABLE 5 (a) and (b), we conclude that there is not enough evidence in the data to disprove the validity of the Gamma-distributed i.i.d. error assumption.

Given the validity of these two main assumptions, theoretical relations $C V\left[X_{t, n}\right]=1 / \sqrt{\pi}=\alpha$ and $C V\left[Y_{t}\right]=1 / \sqrt{\phi}=\beta$ are also examined by computing $\hat{\alpha}_{M L} / \hat{\alpha}$ and $\hat{\beta}_{M L} / \hat{\beta}$, respectively, in the similar fashion as in the simulation study. The results presented in TABLE 4 show that the values of these ratios are very close to 1 in all cases, i.e., $\hat{\alpha}_{M L} / \hat{\alpha}$ for network A, B, and C are 0.978, 1.044, and 0.943, respectively; and $\hat{\beta}_{M L} / \hat{\beta}$ are $0.961,0.989$, and 0.995 , respectively. This indicates that we can interpret the shape parameters ( $\pi$ and $\phi$ ) of the Gamma-Gamma distribution in connection with vehicle-to-vehicle and day-to-day travel time variability $(\alpha$ and $\beta$ ). It also suggests that the CVs of vehicle-to-vehicle and day-to-day travel variability (obtained from the mean and SD measures) can be directly used to construct the Gamma-Gamma PDF of the underlying travel time distribution.

TABLE 5 KS-Test Results for Validating Gamma-distributed i.i.d. Error Assumptions One-sample Kolmogorov-Smirnov (KS) Test Network Network Network

A $\quad$ B $\quad$ C

\section{(a) Test If the Error Term is Gamma-Distributed}

\begin{tabular}{cclccc}
\hline \multirow{2}{*}{$\begin{array}{c}\text { veh-to- } \\
\text { veh }\end{array}$} & $\mathrm{H}_{0}: \gamma$ is from & $-\pi$ estimate $\left(\hat{\pi}_{M L}\right)$ & 48.44 & 50.22 & 23.10 \\
& Gamma $(\pi, 1 / \pi)$ & - KS statistic & 0.039 & 0.039 & 0.032 \\
& & $-p$-value & 0.096 & 0.090 & 0.244 \\
\hline \multirow{2}{*}{$\begin{array}{c}\text { day-to- } \\
\text { day }\end{array}$} & $\mathrm{H}_{0}: \eta$ is from & $-\phi$ estimate $\left(\hat{\phi}_{M L}\right)$ & 1.515 & 3.957 & 2.131 \\
& Gamma $(\phi, 1 / \phi)$ & - KS statistic & 0.057 & 0.066 & 0.029 \\
& & $-p$-value & 0.446 & 0.265 & 0.991 \\
\hline \hline
\end{tabular}

(b) Test If the Error Terms are Independent and Identically Distributed (i.i.d.)

\begin{tabular}{cclccc}
\hline & & $\begin{array}{l}\text { - Number of error groups with } \\
p \text {-value }>0.05\end{array}$ & 219 & 224 & 185 \\
$\begin{array}{c}\text { veh-to- } \\
\text { veh }\end{array}$ & $\begin{array}{c}\mathrm{H}_{0}: \gamma \text { for each }(t, n) \text { is } \\
\text { from Gamma }(\pi, 1 / \pi)\end{array}$ & $\begin{array}{l}\text { - Total number of error groups } \\
-\% \text { of }(t, n) \text { groups not } \\
\text { rejecting } \mathrm{H}_{0} \text { at } 5 \% \text { level }\end{array}$ & 224 & 224 & 224 \\
& & $\begin{array}{l}\text { - Number of } t \text { groups with } p \text { - } \\
\text { value }>0.05\end{array}$ & 14 & 14 & 14 \\
\hline $\begin{array}{c}\text { day-to- } \\
\text { day }\end{array}$ & $\begin{array}{c}\mathrm{H}_{0}: \eta \text { for each } t \text { is } \\
\text { from Gamma }(\phi, 1 / \phi)\end{array}$ & $\begin{array}{l}\text { - Total number of } t \text { groups } \\
-\% \text { of } t \text { groups not rejecting } \\
\mathrm{H}_{0} \text { at } 5 \% \text { level }\end{array}$ & 14 & 14 & 14 \\
& & 100.0 & 100.0 & 100.0 \\
\hline
\end{tabular}

\subsection{Constructing Component Distributions of Gamma-Gamma Distribution}

Based on the estimation results $\left(\hat{\pi}=1 / \hat{\alpha}^{2}, \hat{\phi}=1 / \hat{\beta}^{2}\right.$, and $\left.\hat{\mu}_{1}, \cdots, \hat{\mu}_{T}\right)$ obtained above, we construct the component distributions of the Gamma-Gamma model, namely, $X_{t, n} \sim \operatorname{Gamma}\left(\pi, \mu_{X_{t, n}} / \pi\right)$ and $Y_{t} \sim \operatorname{Gamma}\left(\phi, \mu_{t} / \phi\right)$ to examine the characteristics of each component. The estimated component 
distributions for the three study networks are presented in FIGURE 10. Within each sub-figure, the rotated density plots in (i) represent the day-to-day travel delay distributions at different $t\left(Y_{t}\right)$, where the connected black dots represent the overall mean levels $\left(\mu_{t}\right)$. The three plots on the right represent the (within-day) vehicle-to-vehicle travel delay distributions given a particular daily mean level $\left(y_{t}\right)$, where the values of $y_{t}$ in (ii), (iii), and (iv) are selected to be the $95^{\text {th }}$ percentile, the overall mean $\left(\mu_{t}\right)$, and the $5^{\text {th }}$ percentile of the PDF of $Y_{t}$ at the last time interval, respectively.

From the figure, we can observe the property of constant $\mathrm{CV}$ within each component type; that is, the dispersion of a PDF increases as its mean level increases. Overall, the skewness of day-to-day distributions is much lower than that of vehicle-to-vehicle distributions. This is as expected from the relatively low values of $\hat{\beta}$ in the estimation results, because theoretical relations $\operatorname{Skew}\left[X_{t, n}\right]=$ $2 / \sqrt{\pi}=2 \alpha$ and $\operatorname{Skew}\left[Y_{t}\right]=2 / \sqrt{\phi}=2 \beta$ indicate that the smaller the slope parameter is, the less the distribution is skewed. From the plots in (ii)-(iv), we observe that the shape of the vehicle-to-vehicle distributions varies widely with the daily mean level $\left(y_{t}\right)$. This suggests that, by allowing the mean parameter to fluctuate from day to day, the compound representation can describe a wide spectrum of traffic conditions even within the same time-of-day period (i.e., at the same overall mean level $\mu_{t}$ ).

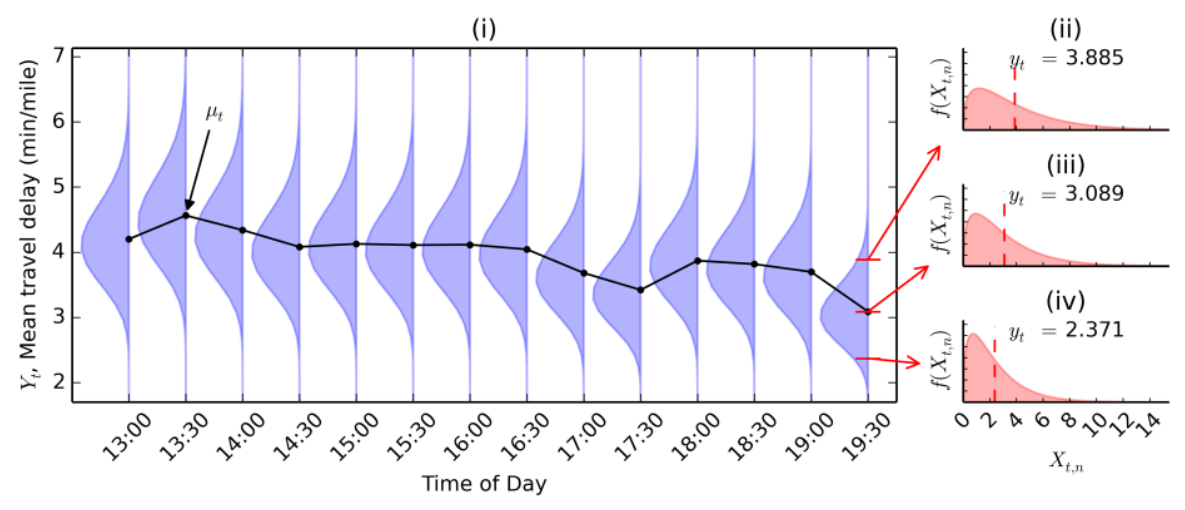

(a)

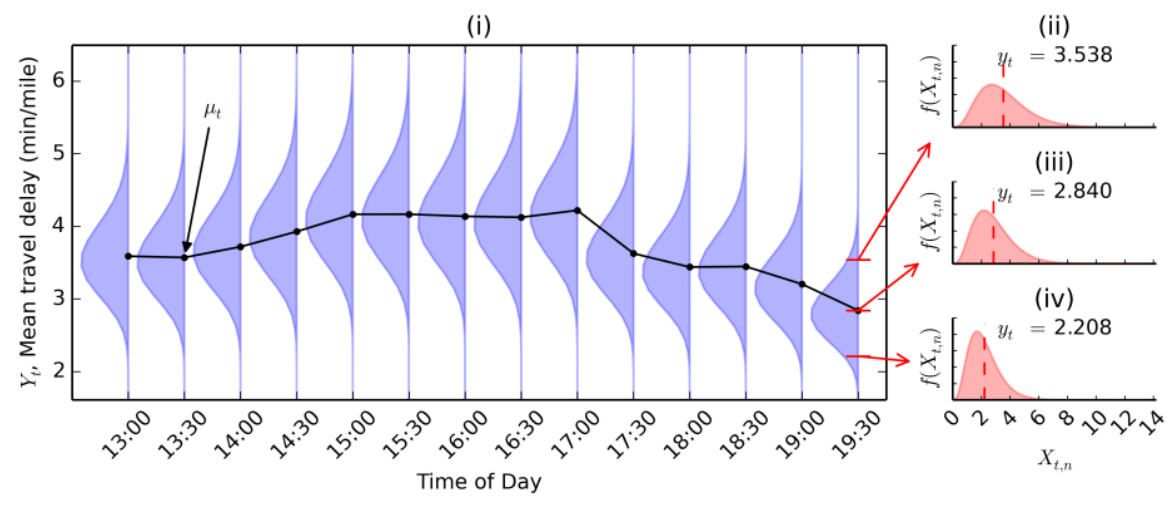

(b) 


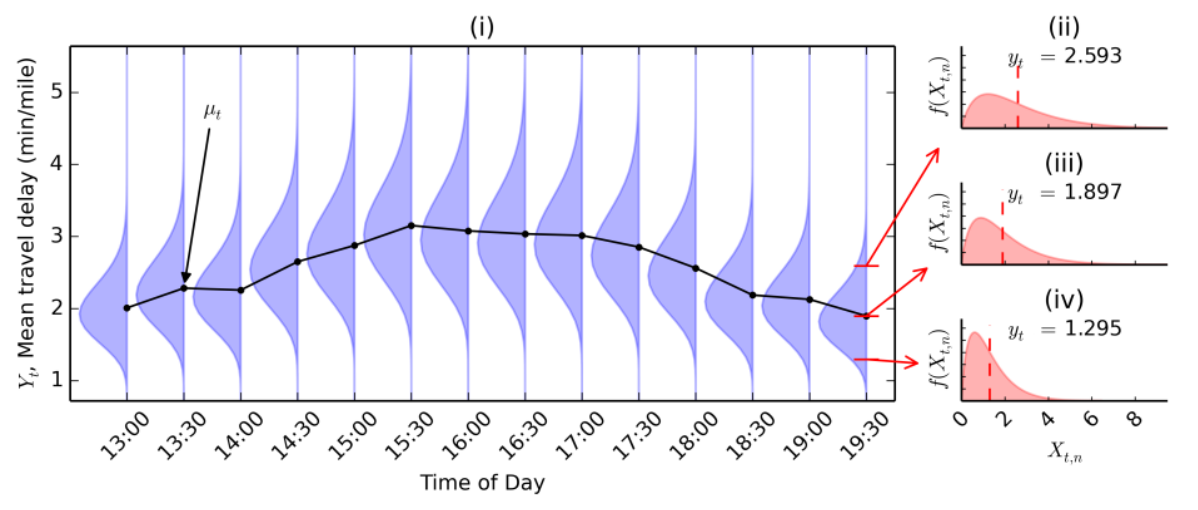

(c)

FIGURE 10 Components of the Gamma-Gamma distribution for (a) network A, (b) network B, and (c) network C: (i) overall mean level $\mu_{t}$ at each $t$ and day-to-day distributions of the mean travel delay given $\boldsymbol{\mu}_{t}, Y_{t} \sim \operatorname{Gamma}\left(\phi, \mu_{t} / \phi\right)$; (ii-iv) vehicle-to-vehicle distributions of travel delay given $\boldsymbol{y}_{t}$, $X_{t, n} \sim \operatorname{Gamma}\left(\pi, \mu_{X_{t, n}} / \pi\right)$, where (ii) $\boldsymbol{y}_{t}=95^{\text {th }}$ percentile, (iii) $\boldsymbol{y}_{t}=\boldsymbol{\mu}_{t}$, and (iv) $\boldsymbol{y}_{t}=5^{\text {th }}$ percentile.

\subsection{Estimating Time-varying Shape Parameters of Extended Gamma-Gamma Model}

A closer look at the mean-SD plots in FIGURE 9 reveals that there exist hysteresis loops in day-to-day mean-SD relationship in networks $B$ and $C$, as can be observed in more detailed plots in FIGURE 11. In Section 3.4, we have introduced CV correction factors $\lambda_{t}^{\alpha}$ and $\lambda_{t}^{\beta}$ to account for such time-of-day dynamics in mean-SD relations. In this section, we calibrate these correction factors using the data exhibiting day-to-day hysteresis patterns to construct the extended Gamma-Gamma model. FIGURE 12 shows the estimated $\lambda_{t}^{\alpha}$ and $\lambda_{t}^{\beta}$ values for each network, where Eqs. (20) and (21) are used for the calculation, respectively. The results show that the values of vehicle-to-vehicle correction factor $\lambda_{t}^{\alpha}$ are close to 1 , where all three networks have the values ranging between 0.9 and 1.1, except for the first two time intervals in network C (see FIGURE 12 (e)). This suggests that vehicle-to-vehicle shape parameter $\pi$ does not have a significant time-of-day variation and could be viewed independent of time $t$, e.g., $\pi_{t}=1 /\left(\lambda_{t}^{\alpha} \alpha\right)^{2} \approx \pi$. Day-to-day correction factor $\lambda_{t}^{\beta}$, on the other hand, shows a clear time-of-day variation, especially in network B and C (see FIGURE 12 (d, f)). Both networks B and C exhibit counterclockwise loops, thereby leading to a wave-like pattern in the $\lambda_{t}^{\beta}$-plot that is similar to the one illustrated in FIGURE 3 (b). This result suggests that, when a hysteresis exists in the mean-SD relationship, day-today shape parameter $\phi$ is no longer time-invariant and it might be necessary to adjust it through $\phi_{t}=1 /\left(\lambda_{t}^{\beta} \beta\right)^{2}$ to produce a more realistic travel time distribution. It is, however, not known how sensitive the resulting travel time distribution would be to such deviations of actual $\phi_{t}$ from static $\phi$. In the next section, we will examine this aspect by comparing the performances of the Gamma-Gamma distributions with and without time-varying shape parameters. 


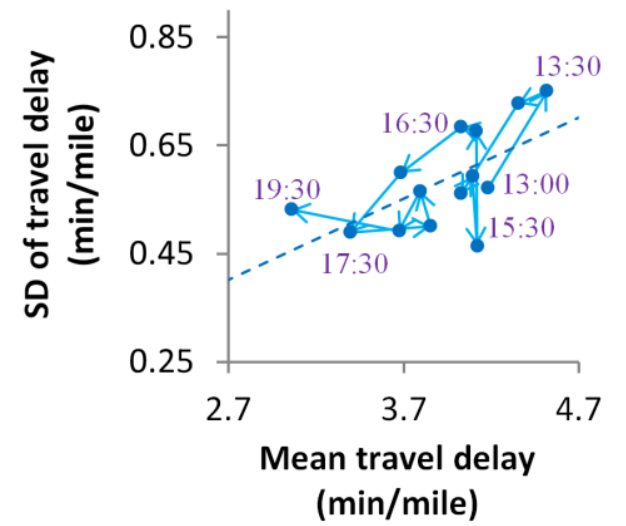

(a)

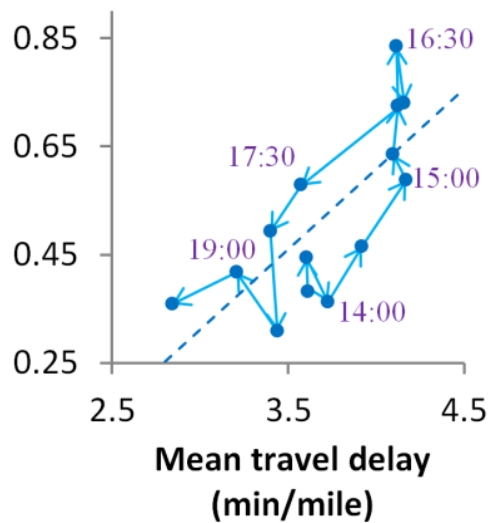

(b)

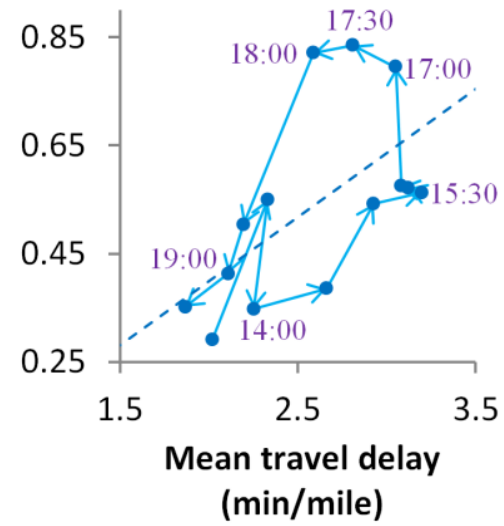

(c)

FIGURE 11 Relationship between day-to-day mean and SD of travel delay per unit distance during PM period (1 PM - 8 PM): (a) network A, (b) network B, and (c) network $C$.

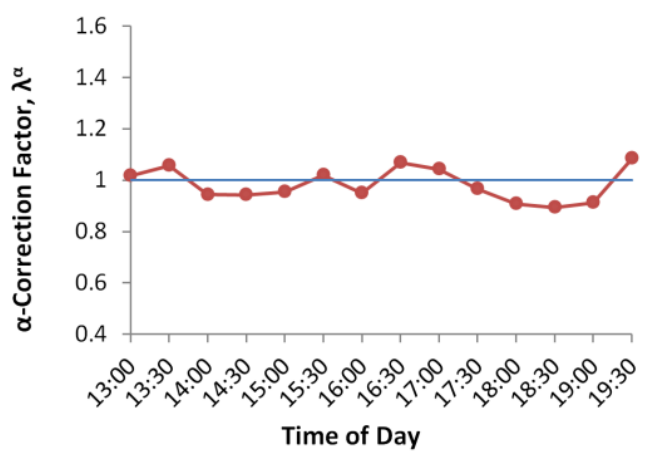

(a)

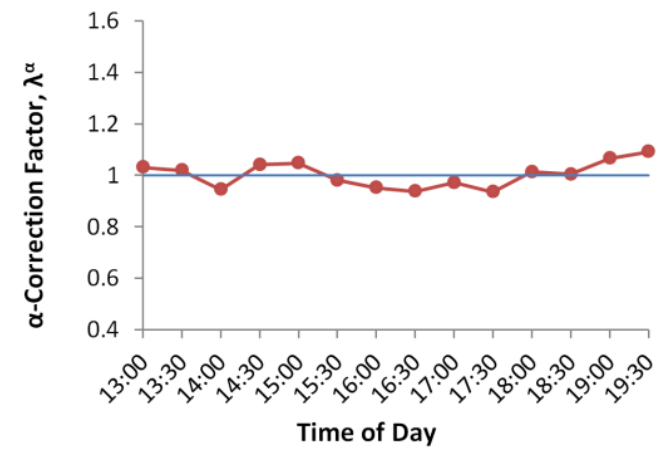

(c)

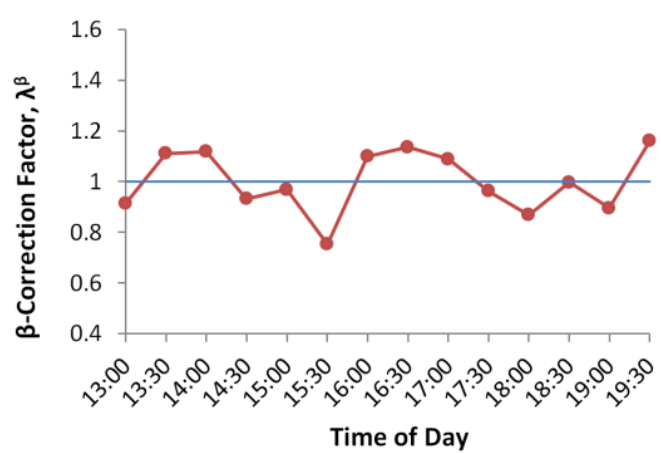

(b)

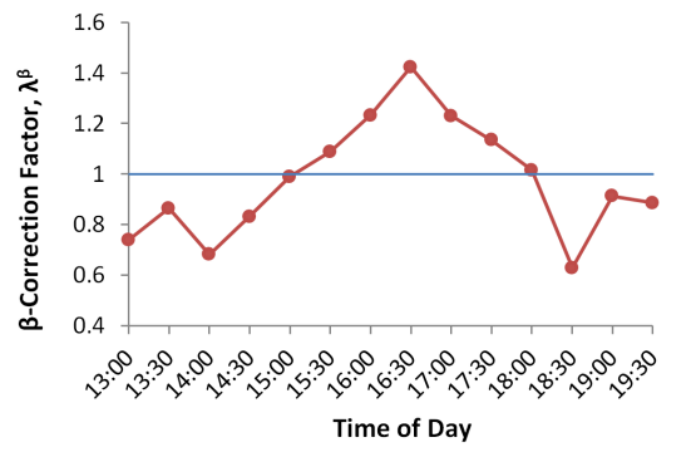

(d) 


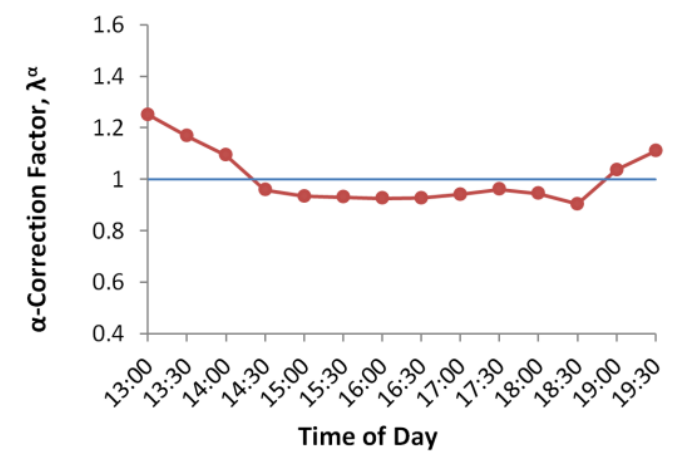

(e)

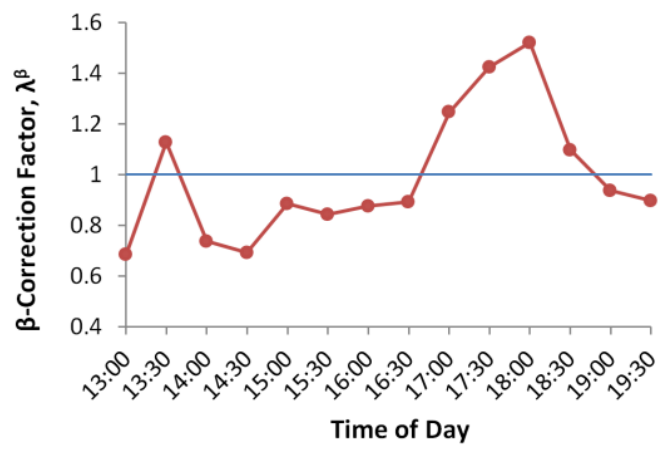

(f)

FIGURE 12 Estimates of CV correction factors in the extended Gamma-Gamma model for (a,b) network A, (c,d) network B, and (e,f) network C: (left) vehicle-to-vehicle CV correction factor, $\lambda_{t}^{\alpha}$, and (right) day-to-day $\mathrm{CV}$ correction factor, $\lambda_{t}^{\beta}$.

\subsection{Evaluating Performance of Gamma-Gamma Distribution}

Following the procedure described in Section 4.4, we compare the performance of the Gamma-Gamma distribution with that of Gamma and Lognormal distributions using the real-world probe data from networks A, B, and C. In addition to these three models, we also examine the Gamma-Gamma distribution with time-varying shape parameters, denoted by Gamma-Gamma_t, where the parameters are estimated using $\hat{\pi}_{t}=1 /\left(\hat{\lambda}_{t}^{\alpha} \hat{\alpha}\right)^{2}$ and $\hat{\phi}_{t}=1 /\left(\hat{\lambda}_{t}^{\beta} \hat{\beta}\right)^{2}$ given the CV correction factors obtained in the previous section. FIGURE 13 presents the overall and time-of-day (relative) performance of each model through $\triangle A I C_{j}$ and $L L_{j}-\overline{L L}$, respectively, in the same manner as FIGURE 6.

The estimation results of $\triangle A I C_{j}$ show that the Gamma-Gamma distribution is favored over the other three models in all three networks (i.e., $\triangle A I C_{j}=0$ ). The Gamma-Gamma_t is found to be the second best model in all three networks, with relatively small differences from the Gamma-Gamma model (i.e., $\Delta A I C_{j}=28.97,18.14$, and 83.71 for networks A, B, and C, respectively). The performance of the Gamma and Lognormal distributions largely depends on the study network. In networks A and B, the Gamma distribution performs better than the Lognormal distribution, where the Lognormal shows very low goodness-of-fit (i.e., $\triangle A I C_{j}=2354.39$ and 1011.75). In network $\mathrm{C}$, however, the Lognormal performs better than the Gamma distribution.

From the results of the relative log-likelihood, we observe that the overall performance differences estimated by $\triangle A I C_{j}$ are maintained over time $t$ in networks $\mathrm{A}$ and $\mathrm{B}$, whereas the relative performance of the models varies widely with the time interval in network $\mathrm{C}$. Another observation is that the rankings between the Gamma-Gamma and Gamma-Gamma_t are now reversed; that is, the GammaGamma_t shows slightly higher log-likelihood values in most time intervals. This suggests that, while the Gamma-Gamma_t is largely penalized by the AIC due to its extra parameters $(k=3 \times T=42)$, the actual fit without factoring in the penalty tends to be better under the Gamma-Gamma_t than under the GammaGamma model. This is because the Gamma-Gamma_t can adapt to changes in data more flexibly. However, such flexibility does not always lead to a better performance as the Gamma-Gamma_t shows lower goodness-of-fit than the Gamma-Gamma during the beginning and end periods in FIGURE 13(c). 
FIGURE 14 provides some examples of detailed histograms of travel delay data, presented along with the fitted density curves. For each network, the peak time interval where the mean travel delay is the highest was selected for the plot. The selected peak intervals are 13:30, 15:00, and 15:30 for network A, B, and $\mathrm{C}$, respectively. The patterns in FIGURE 14 are consistent with those in FIGURE 7 from the simulation study in that the Gamma-Gamma family effectively adapts to the underlying travel delay data. The capability to model a wide range of traffic conditions is an important feature of the Gamma-Gamma model; in the context of travel time reliability analysis, it can offer a unifying framework for describing, comparing, and assessing travel time distributions from different networks and time periods.

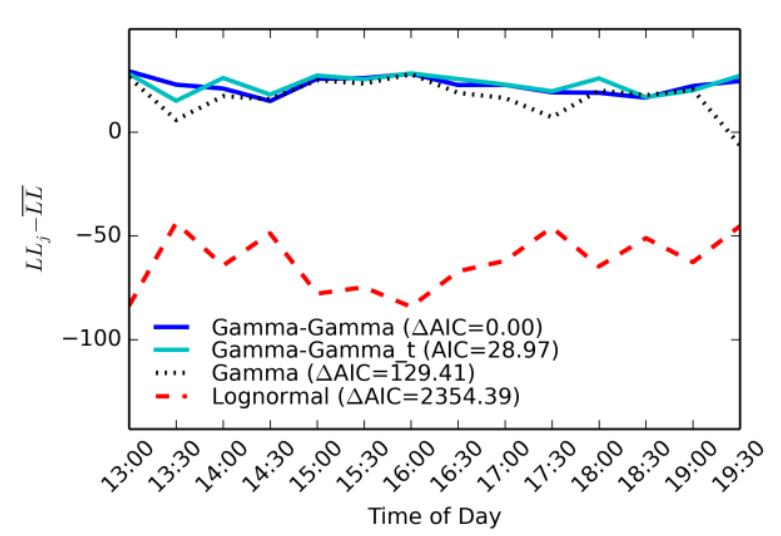

(a)

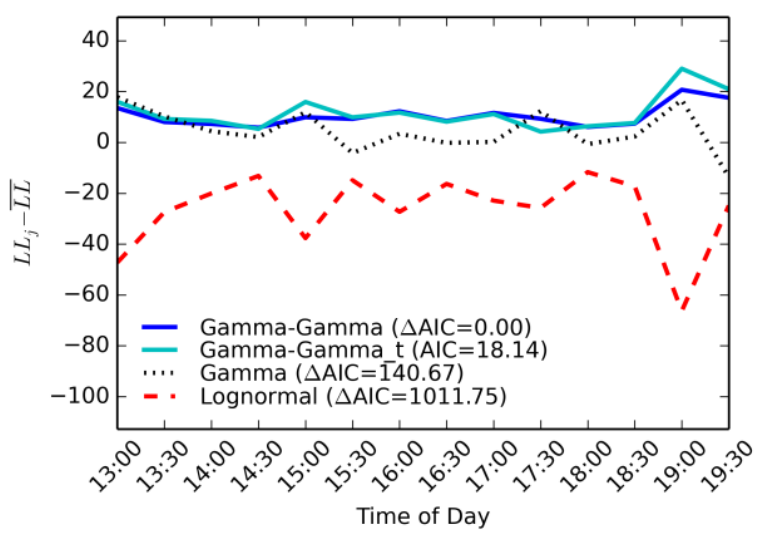

(b)

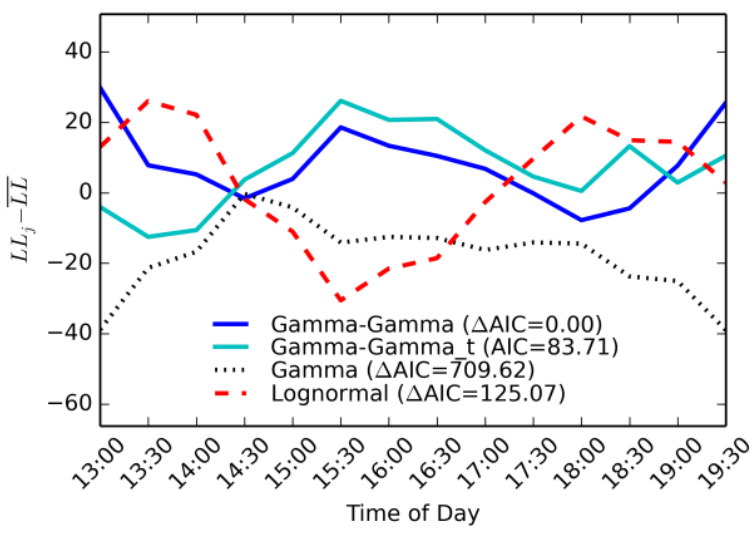

(c)

FIGURE 13 Relative values of log-likelihood under Gamma-Gamma, Gamma-Gamma_t, Gamma and Lognormal distributions fitted to travel delay data 


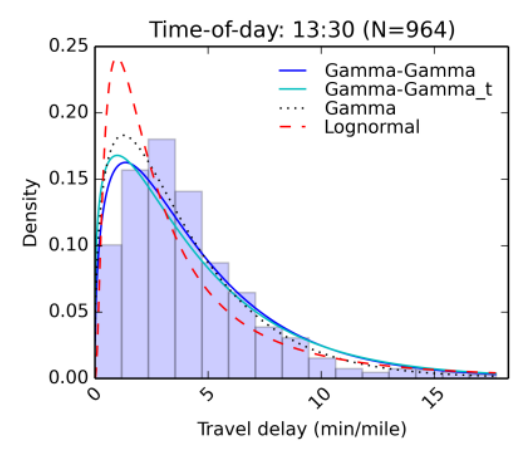

(a)

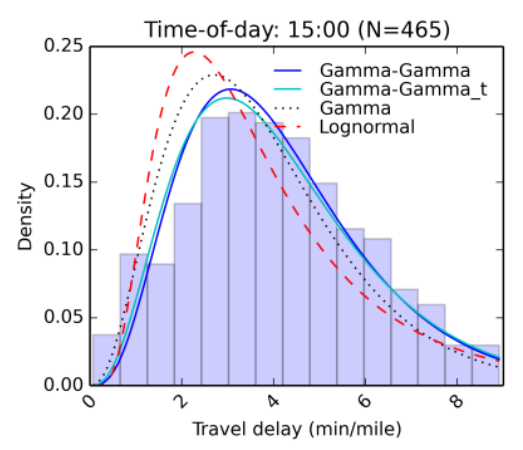

(b)

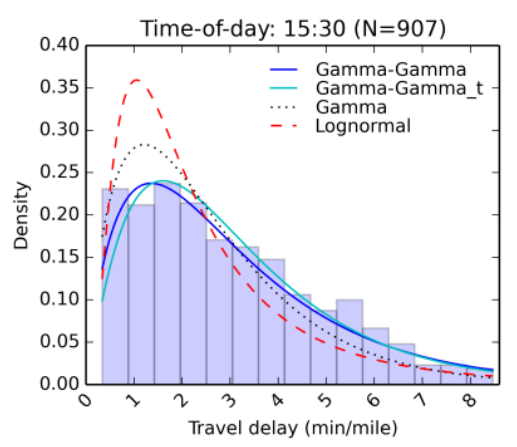

(c)

FIGURE 14 Travel delay distribution (across vehicles and days) and the fitted density curves; data are from the actual vehicle trajectories from (a) network $A$ at 13:30, (b) network $B$ at 15:00, and (c) network $C$ at 15:30.

Next, we take a closer look at the performance difference between the Gamma-Gamma and GammaGamma_t distributions. The Gamma-Gamma_t adjusts its shape parameters through CV correction factors $\lambda_{t}^{\alpha}$ and $\lambda_{t}^{\beta}$ to account for deviations from the global vehicle-to-vehicle and day-to-day variability trends captured by $\alpha$ and $\beta$. In FIGURE 15, we compare the Gamma-Gamma and Gamma-Gamma_t distributions fitted to the data from network $C$, focusing on two time intervals 14:30 and 18:00, where the value of $\lambda_{t}^{\beta}$ deviated from 1 the most due to the hysteresis loop in the day-to-day mean-SD relationship (see FIGURE 11 (c) ). In FIGURE 12 (f), the value of $\lambda_{t}^{\beta}$ is found to be 0.69 at 14:30 and 1.52 at 18:00, yielding adjusted slope $\hat{\lambda}_{t}^{\beta} \hat{\beta}$ of 0.14 and 0.32 , respectively. The associated time-varying shape parameter for the GammaGamma_t, $\hat{\phi}_{t}=1 /\left(\hat{\lambda}_{t}^{\beta} \hat{\beta}\right)^{2}$, is therefore 47.63 at $14: 30$ and 9.81 at 18:00. The value of original slope $\hat{\beta}$ is 0.21 as shown in TABLE 4 and the associated shape parameter is 22.68 from $\hat{\phi}=1 / \hat{\beta}^{2}$. The results in FIGURE 15 show that there exists a distinguishable difference between these two distributions in both cases, which might have led the performance difference in the log-likelihood values in FIGURE 13 (c) - the log-likelihood under the Gamma-Gamma_t model is higher than that under the Gamma-Gamma model in both 14:30 and 18:00. However, the performance gain by switching from the Gamma-Gamma model to the Gamma-Gamma_t model appears to be rather small given the large number of additional parameters, as also revealed by the $\triangle A I C_{j}$ results. This limitation could be overcome if we could find a method to identify or predict the values of the correction factors at a particular time interval in a more efficient way. 


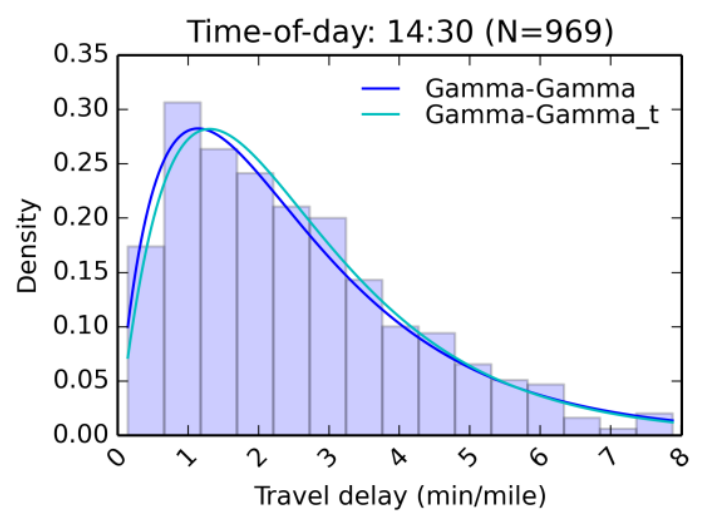

(a)

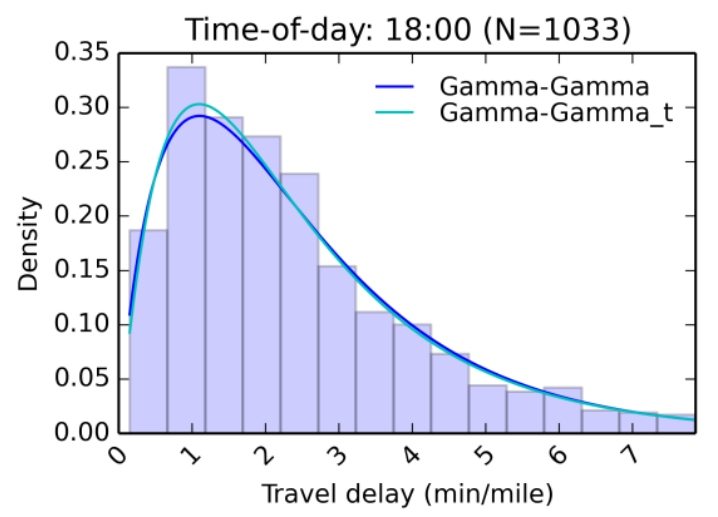

(b)

FIGURE 15 Effect of time-varying shape parameters (Gamma-Gamma vs. Gamma-Gamma_t); histogram and fitted curves of travel delay data from network $C$ : (a) 14:30 and (b) 18:00.

\section{APPLICATIONS}

For travel time reliability analysis, the Gamma-Gamma distribution can be used as a tool for providing a complete representation of the individual travel delay distribution-across vehicles and days - at a particular time interval. By knowing the CVs of vehicle-to-vehicle and day-to-day distributions and the time-dependent mean levels, one can easily construct the Gamma-Gamma distribution to obtain any reliability measure. It is beneficial to have a probability density function along with descriptive statistics as it provides better insight into the underlying distribution that is necessary for generalizing beyond a particular experiment or sample. In addition, the model can be effectively used for simulation-based reliability analysis as the straightforward interpretation of the model parameters - two shape parameters represent the inverse of the square of the CVs of vehicle-to-vehicle and day-to-day distributions-allows one to easily adjust and change parameter values to examine travel time distributions under different types and levels of variability.

An important application of the Gamma-Gamma modeling framework is in the evaluation of a policy or project that affects travel time reliability in a given network. The compound representation that both distinguishes and connects vehicle-to-vehicle and day-to-day variability allows policy makers to evaluate the effect of a given policy on each type of variability separately as well as on the overall travel time distribution as a whole. One particular application area is weather-responsive traffic management, which aims to mitigate weather-related congestion on days with severe weather by deploying various advisory and control strategies (Kim et al., 2013a; Mahmassani et al., 2012b). From the travel time reliability perspective, such strategies are to reduce day-to-day variability and their effectiveness in improving reliability can be systematically analyzed using the proposed model.

In addition to its application to reliability assessment within a given network, the Gamma-Gamma model can be used as a unifying framework for analyzing and comparing travel time reliability across different networks. As observed in the estimation results using the simulated and actual data, the compound representation of vehicle-to-vehicle and day-to-day component distributions allows the final compound probability distribution to describe a wide spectrum of traffic conditions, by flexibly adapting its shape to the observed data. This makes the Gamma-Gamma distribution suitable for modeling travel time data from various places and times - from arterials to freeways and from off-peaks to rush hours- 
and allows a systematic comparison of the underlying travel time distributions through the estimated Gamma-Gamma model parameters.

\section{CONCLUSION}

This paper proposes a compound representation for describing both vehicle-to-vehicle and day-to-day variability in modeling travel time reliability. The proposed model is based on the product of two Gamma distributions: one represents vehicle-to-vehicle variations in individual travel delay measures and the other represents fluctuations in day-to-day mean level. Starting from the observation that there exist linear or near-linear relationships between mean and SD of travel time per unit distance for both vehicle-tovehicle and day-to-day distributions, we build a multiplicative error model to describe each of these two types of distributions accounting for those linear relationships. The study uses travel delay per unit distance, obtained by subtracting the minimum travel time per unit distance from travel time per unit distance, as a surrogate for travel time for the mathematical tractability of the model derivation. Combining two multiplicative models yields a compound distribution (or product distribution) of two Gamma variables, known as Gamma-Gamma model, with its PDF available in a closed form. The Gamma-Gamma model involves three parameters: $\pi, \phi$, and $\mu_{t}$, which have the following interpretation in our context:

- The shape $\pi$ characterizes vehicle-to-vehicle variability as $1 / \sqrt{\pi}$ represents the CV of individual travel delay across vehicles;

- The shape $\phi$ characterizes day-to-day variability as $1 / \sqrt{\phi}$ represents the $\mathrm{CV}$ of daily mean level of travel delay across different days; and

- The mean $\mu_{t}$ represents an underlying true mean of travel delay at a given time interval $t$.

An extension of the basic model form was also introduced to account for deviations of mean-SD relationships from the linearity assumption. By applying time-varying correction factors to the two shape parameters, the extended Gamma-Gamma model can now capture time-of-day variations in mean-SD relations through time-varying shape parameters $\pi_{t}$ and $\phi_{t}$, in addition to the existing time-of-day congestion levels captured by $\mu_{t}$.

The model parameters are estimated and the underlying assumptions are validated using both simulated and actual vehicle trajectory data. From the analysis results, it is found that the relationship between mean and SD of travel delay per unit distance can be adequately represented by a linear (proportional) model in both vehicle-to-vehicle and day-to-day distributions. The performance of the Gamma-Gamma distribution - in both basic and extended forms - is tested and compared with traditional Gamma and Lognormal distributions. The results show that the basic Gamma-Gamma distribution is favored over the other three models, suggesting that the Gamma-Gamma model provides a flexible yet simple model to describe travel delay distributions for different time intervals and for different parts of the network. The main advantage of the Gamma-Gamma model is its ability to recognize different variability dimensions reflected in travel time data and a clear physical meaning of its parameters in connection with vehicle-to-vehicle and day-to-day variability. As such, the model provides a systematic way of quantifying, comparing, and assessing different types of variability, which is important in understanding travel time characteristics and evaluating various transportation measures that affect reliability. 


\section{ACKNOWLEDGMENT}

This paper is based on research conducted under the Strategic Highway Research Program SHRP-2 project L04 "Incorporating Reliability Performance Measures in Operations and Planning Modeling Tools", as well as under National Science Foundation Grant No. CMS 0928577 "Toward More Reliable Mobility: Improved Decision Support Tools for Transportation Systems". The authors would like to acknowledge the support of the Northwestern University Transportation Center. The work presented here reflects valuable comments obtained from the SHRP-2 project Technical Expert Task Group, as well as various collaborators at Northwestern. The authors are especially grateful to William (Bill) Hyman, SHRP-2 reliability program manager and Stephen Andrle, SHRP-2 capacity program manager, for their continued support and encouragement throughout this effort. The paper has greatly benefited from the comments and suggestions of two anonymous reviewers, particularly in the analysis conducted as part of the validation section, and in the extension to consider time-varying conditions. The authors of course remain solely responsible for the content of this paper. 


\section{REFERENCES}

Abraham, D.A., 2003. Signal excess in K-distributed reverberation. IEEE Journal of Oceanic Engineering 28, 526-536. doi:10.1109/JOE.2003.816675

Al-Ahmadi, S., Yanikomeroglu, H., 2010. On the approximation of the generalized-K distribution by a gamma distribution for modeling composite fading channels. IEEE Transactions on Wireless Communications 9, 706-713. doi:10.1109/TWC.2010.02.081266

Al-Deek, H., Emam, E.B., 2006. New Methodology for Estimating Reliability in Transportation Networks with Degraded Link Capacities. Journal of Intelligent Transportation Systems 10, 117-129. doi:10.1080/15472450600793586

Al-Habash, M.A., Andrews, L.C., Phillips, R.L., 2001. Mathematical model for the irradiance probability density function of a laser beam propagating through turbulent media. Opt. Eng 40, 1554-1562. doi:10.1117/1.1386641

Arroyo, S., Kornhauser, A.L., 2005. Modeling Travel Time Distributions on a Road Network, in: Proceedings of the 84th Annual Meeting of the Transportation Research Board, Washington, D.C. Presented at the Transportation Research Board 84th Annual Meeting.

Blacknell, D., 1994. Comparison of parameter estimators for K-distribution. Radar, Sonar and Navigation, IEE Proceedings 141, 45-52. doi:10.1049/ip-rsn:19949885

Brownlees, C.T., Cipollini, F., Gallo, G.M., 2011. Multiplicative Error Models (SSRN Scholarly Paper No. ID 1852285). Social Science Research Network, Rochester, NY.

Cambridge Systematics, Inc. et al., 2010. Analytical Procedures for Determining the Impacts of Reliability Mitigation Strategies, NCHRP SHRP 2 Project L03.

Chatzidiamantis, N.D., Karagiannidis, G.K., 2009. On the Distribution of the Sum of Gamma-Gamma Variates and Applications in RF and Optical Wireless Communications (arXiv e-print No. 0905.1305).

Eisenhauer, J.G., 2003. Regression through the Origin. Teaching Statistics 25, 76-80. doi:10.1111/14679639.00136

Engle, R., 2002. New frontiers for arch models. Journal of Applied Econometrics 17, 425-446. doi:10.1002/jae.683

Engle, R.F., Gallo, G.M., 2006. A multiple indicators model for volatility using intra-daily data. Journal of Econometrics 131, 3-27. doi:10.1016/j.jeconom.2005.01.018

Fosgerau, M., 2010. On the Relation Between the Mean and Variance of Delay in Dynamic Queues with Random Capacity and Demand. Journal of Economic Dynamics and Control 34, 598-603. doi:10.1016/j.jedc.2009.12.002

Fosgerau, M., Fukuda, D., 2012. Valuing Travel Time Variability: Characteristics of the Travel Time Distribution on an Urban Road. Transportation Research Part C: Emerging Technologies 24, 83101. doi:10.1016/j.trc.2012.02.008

Gayah, V.V., Dixit, V.V., Guler, S.I., 2013. Relationship between mean and day-to-day variation in travel time in urban networks. EURO J Transp Logist 1-17. doi:10.1007/s13676-013-0032-2

Gradshteyn, I.S., Ryzhik, I.M., Jeffrey, A., Zwillinger, D., 2007. Table of integrals, series and products. Academic, Oxford.

Gu, M., Abraham, D.A., 2001. Using McDaniel's model to represent non-Rayleigh reverberation. IEEE Journal of Oceanic Engineering 26, 348-357. doi:10.1109/48.946509

Guo, F., Rakha, H., Park, S., 2010. Multistate Model for Travel Time Reliability. Transportation Research Record: Journal of the Transportation Research Board 2188, 46-54. doi:10.3141/2188-06

Herman, R., Lam, T.C., 1974. Trip Time Characteristics of Journeys to and from Work. Presented at the Transportation and Traffic Theory, Proceedings. 
Jakeman, E., Pusey, P.N., 1976. A model for non-Rayleigh sea echo. IEEE Transactions on Antennas and Propagation 24, 806-814. doi:10.1109/TAP.1976.1141451

Jiang, L., Mahmassani, H.S., Zhang, K., 2011. Congestion Pricing, Heterogeneous Users, and Travel Time Reliability. Transportation Research Record: Journal of the Transportation Research Board 2254, 58-67. doi:10.3141/2254-07

Jones, E.G., Mahmassani, H.S., Herman, R., Walton, C.M., 1989. Travel Time Variability in a Commuting Corridor: Implications for Electronic Route Guidance. Presented at the First International Conference on Applications of Advanced Technologies in Transportation Engineering.

Kazeminia, M., Mehrjoo, M., 2013. A New Method for Maximum Likelihood Parameter Estimation of Gamma-Gamma Distribution. Journal of Lightwave Technology 31, 1347-1353. doi:10.1109/JLT.2013.2246858

Kim, J., 2014. Travel Time Reliability of Traffic Networks: Characterization, Modeling, and Scenario-based Simulation (Ph.D. Dissertation). Northwestern University, Evanston, IL.

Kim, J., Mahmassani, H., Alfelor, R., Chen, Y., Hou, T., Jiang, L., Saberi, M., Verbas, Ö., Zockaie, A., $2013 a$. Implementation and Evaluation of Weather-Responsive Traffic Management Strategies: Insight from Different Networks. Transportation Research Record: Journal of the Transportation Research Board 2396, 93-106. doi:10.3141/2396-11

Kim, J., Mahmassani, H.S., 2014. A Finite Mixture Model of Vehicle-to-Vehicle and Day-to-Day Variability of Traffic Network Travel Times. Transportation Research Part C: Emerging Technologies 46, 8397. doi:10.1016/j.trc.2014.05.011

Kim, J., Mahmassani, H., Vovsha, P., Stogios, Y., Dong, J., 2013b. Scenario-Based Approach to Analysis of Travel Time Reliability with Traffic Simulation Models. Transportation Research Record: Journal of the Transportation Research Board 2391, 56-68. doi:10.3141/2391-06

Lewinski, D., 1983. Nonstationary probabilistic target and clutter scattering models. IEEE Transactions on Antennas and Propagation 31, 490-498. doi:10.1109/TAP.1983.1143067

MacCullagh, P., Nelder, J.A., 1989. Generalized Linear Models. CRC Press.

Mahmassani, H.S., Hou, T., Dong, J., 2012a. Characterizing Travel Time Variability in Vehicular Traffic Networks: Deriving a Robust Relation for Reliability Analysis. Transportation Research Record: Journal of the Transportation Research Board 2315, 141-152. doi:10.3141/2315-15

Mahmassani, H.S., Hou, T., Saberi, M., 2013a. Connecting Networkwide Travel Time Reliability and the Network Fundamental Diagram of Traffic Flow. Transportation Research Record: Journal of the Transportation Research Board 2391, 80-91. doi:10.3141/2391-08

Mahmassani, H.S., Kim, J., Hou, T., Zockaie, A., Saberi, M., Jiang, L., Verbas, O., Cheng, S., Chen, Y., Hass, R., 2012b. Implementation and Evaluation of Weather Responsive Traffic Estimation and Prediction System (Final Report No. FHWA-JPO-12-055). FHWA, U.S. Department of Transportation.

Mahmassani, H.S., Kim, J., Stogios, Y., Currie, K., Vovsha, P., 2013b. Incorporating Reliability Performance Measures in Operations and Planning Modeling Tools (SHRP2 Project L04 Final Report No. S2L04-RR-1).

Mahmassani, H.S., Mudge, R., Hou, T., Kim, J., 2012c. Use of Mobile Data for Weather-Responsive Traffic Management Models (Final Report No. FHWA-JPO-13-003). FHWA, U.S. Department of Transportation.

Mahmassani, H.S., Sbayti, H., 2009. DYNASMART-P Version 1.6 User's Guide.

May, A.D., Bonsall, P.W., Marler, N.W., 1989. Travel Time Variability of a Group of Car Commuters in North London (Monograph No. Working Paper 277). University of Leeds.

Mazloumi, E., Currie, G., Rose, G., 2010. Using GPS Data to Gain Insight into Public Transport Travel Time Variability. Journal of Transportation Engineering 136, 623-631. doi:10.1061/(ASCE)TE.19435436.0000126 
Noland, R.B., Polak, J.W., 2002. Travel time variability: A review of theoretical and empirical issues. Transport Reviews 22, 39-54. doi:10.1080/01441640010022456

Peer, S., Koopmans, C.C., Verhoef, E.T., 2012. Prediction of travel time variability for cost-benefit analysis. Transportation Research Part A: Policy and Practice 46, 79-90. doi:10.1016/j.tra.2011.09.016

PeMS [WWW Document], n.d. . Caltrans Performance Measurement System. URL http://pems.dot.ca.gov/ (accessed 4.20.13).

Polus, A., 1979. A study of travel time and reliability on arterial routes. Transportation 8, 141-151. doi:10.1007/BF00167196

Pu, W., 2011. Analytic Relationships Between Travel Time Reliability Measures. Transportation Research Record: Journal of the Transportation Research Board 2254, 122-130. doi:10.3141/2254-13

Rakha, H., El-Shawarby, I., Arafeh, M., Dion, F., 2006. Estimating Path Travel-Time Reliability, in: IEEE Intelligent Transportation Systems Conference, 2006. ITSC'06. Presented at the IEEE Intelligent Transportation Systems Conference, 2006. ITSC '06, pp. 236-241. doi:10.1109/ITSC.2006.1706748

Redding, N.J., 1999. Estimating the Parameters of the K Distribution in the Intensity Domain.

Richardson, A.J., Taylor, M., 1978. Travel Time Variability on Commuter Journeys. High Speed Ground Transportation Journal 12.

Shankar, P.M., 2004. Error Rates in Generalized Shadowed Fading Channels. Wireless Personal Communications 28, 233-238. doi:10.1023/B:wire.0000032253.68423.86

Susilawati, S., Taylor, M.A.P., Somenahalli, S.V.C., 2011. Distributions of travel time variability on urban roads. Journal of Advanced Transportation. doi:10.1002/atr.192

Taylor, M., 1982. Travel Time Variability-The Case of Two Public Modes. Transportation Science 16, 507-521. doi:10.1287/trsc.16.4.507

Taylor, M.A.P., Susilawati, 2012. Modelling Travel Time Reliability with the Burr Distribution. Procedia Social and Behavioral Sciences 54, 75-83. doi:10.1016/j.sbspro.2012.09.727

Teich, M.C., Diament, P., 1989. Multiply stochastic representations for K distributions and their Poisson transforms. J. Opt. Soc. Am. A 6, 80-91. doi:10.1364/JOSAA.6.000080

Verbas, I., Mahmassani, H., Zhang, K., 2011. Time-Dependent Origin-Destination Demand Estimation. Transportation Research Record: Journal of the Transportation Research Board 2263, 45-56. doi:10.3141/2263-06

Wang, N., Cheng, J., 2010. Moment-based estimation for the shape parameters of the Gamma-Gamma atmospheric turbulence model. Opt. Express 18, 12824-12831. doi:10.1364/OE.18.012824

Yildirimoglu, M., Koymans, A., Geroliminis, N., 2013. Exploring the Properties of Mean-Variance Relations in Freeway Travel Times, in: Proceedings of the 92nd Annual Meeting of the Transportation Research Board, Washington, D.C. Presented at the Transportation Research Board 92nd Annual Meeting, Washington, D.C.

Abraham, D.A., 2003. Signal excess in K-distributed reverberation. IEEE Journal of Oceanic Engineering 28, 526-536. doi:10.1109/JOE.2003.816675

Al-Ahmadi, S., Yanikomeroglu, H., 2010. On the approximation of the generalized-K distribution by a gamma distribution for modeling composite fading channels. IEEE Transactions on Wireless Communications 9, 706-713. doi:10.1109/TWC.2010.02.081266

Al-Deek, H., Emam, E.B., 2006. New Methodology for Estimating Reliability in Transportation Networks with Degraded Link Capacities. Journal of Intelligent Transportation Systems 10, 117-129. doi:10.1080/15472450600793586

Al-Habash, M.A., Andrews, L.C., Phillips, R.L., 2001. Mathematical model for the irradiance probability density function of a laser beam propagating through turbulent media. Opt. Eng 40, 1554-1562. doi:10.1117/1.1386641 
Arroyo, S., Kornhauser, A.L., 2005. Modeling Travel Time Distributions on a Road Network, in:

Proceedings of the 84th Annual Meeting of the Transportation Research Board, Washington, D.C. Presented at the Transportation Research Board 84th Annual Meeting.

Blacknell, D., 1994. Comparison of parameter estimators for K-distribution. Radar, Sonar and Navigation, IEE Proceedings 141, 45-52. doi:10.1049/ip-rsn:19949885

Brownlees, C.T., Cipollini, F., Gallo, G.M., 2011. Multiplicative Error Models (SSRN Scholarly Paper No. ID 1852285). Social Science Research Network, Rochester, NY.

Cambridge Systematics, Inc. et al., 2010. Analytical Procedures for Determining the Impacts of Reliability Mitigation Strategies, NCHRP SHRP 2 Project L03.

Chatzidiamantis, N.D., Karagiannidis, G.K., 2009. On the Distribution of the Sum of Gamma-Gamma Variates and Applications in RF and Optical Wireless Communications (arXiv e-print No. 0905.1305).

Eisenhauer, J.G., 2003. Regression through the Origin. Teaching Statistics 25, 76-80. doi:10.1111/14679639.00136

Engle, R., 2002. New frontiers for arch models. Journal of Applied Econometrics 17, 425-446. doi:10.1002/jae.683

Engle, R.F., Gallo, G.M., 2006. A multiple indicators model for volatility using intra-daily data. Journal of Econometrics 131, 3-27. doi:10.1016/j.jeconom.2005.01.018

Fosgerau, M., 2010. On the Relation Between the Mean and Variance of Delay in Dynamic Queues with Random Capacity and Demand. Journal of Economic Dynamics and Control 34, 598-603. doi:10.1016/j.jedc.2009.12.002

Fosgerau, M., Fukuda, D., 2012. Valuing Travel Time Variability: Characteristics of the Travel Time Distribution on an Urban Road. Transportation Research Part C: Emerging Technologies 24, 83101. doi:10.1016/j.trc.2012.02.008

Gayah, V.V., Dixit, V.V., Guler, S.I., 2013. Relationship between mean and day-to-day variation in travel time in urban networks. EURO J Transp Logist 1-17. doi:10.1007/s13676-013-0032-2

Gradshteyn, I.S., Ryzhik, I.M., Jeffrey, A., Zwillinger, D., 2007. Table of integrals, series and products. Academic, Oxford.

Gu, M., Abraham, D.A., 2001. Using McDaniel's model to represent non-Rayleigh reverberation. IEEE Journal of Oceanic Engineering 26, 348-357. doi:10.1109/48.946509

Guo, F., Rakha, H., Park, S., 2010. Multistate Model for Travel Time Reliability. Transportation Research Record: Journal of the Transportation Research Board 2188, 46-54. doi:10.3141/2188-06

Herman, R., Lam, T.C., 1974. Trip Time Characteristics of Journeys to and from Work. Presented at the Transportation and Traffic Theory, Proceedings.

Jakeman, E., Pusey, P.N., 1976. A model for non-Rayleigh sea echo. IEEE Transactions on Antennas and Propagation 24, 806-814. doi:10.1109/TAP.1976.1141451

Jiang, L., Mahmassani, H.S., Zhang, K., 2011. Congestion Pricing, Heterogeneous Users, and Travel Time Reliability. Transportation Research Record: Journal of the Transportation Research Board 2254, 58-67. doi:10.3141/2254-07

Jones, E.G., Mahmassani, H.S., Herman, R., Walton, C.M., 1989. Travel Time Variability in a Commuting Corridor: Implications for Electronic Route Guidance. Presented at the First International Conference on Applications of Advanced Technologies in Transportation Engineering.

Kazeminia, M., Mehrjoo, M., 2013. A New Method for Maximum Likelihood Parameter Estimation of Gamma-Gamma Distribution. Journal of Lightwave Technology 31, 1347-1353. doi:10.1109/JLT.2013.2246858

Kim, J., 2014. Travel Time Reliability of Traffic Networks: Characterization, Modeling, and Scenario-based Simulation (Ph.D. Dissertation). Northwestern University, Evanston, IL. 
Kim, J., Mahmassani, H., Alfelor, R., Chen, Y., Hou, T., Jiang, L., Saberi, M., Verbas, Ö., Zockaie, A., $2013 a$. Implementation and Evaluation of Weather-Responsive Traffic Management Strategies: Insight from Different Networks. Transportation Research Record: Journal of the Transportation Research Board 2396, 93-106. doi:10.3141/2396-11

Kim, J., Mahmassani, H.S., 2014. A Finite Mixture Model of Vehicle-to-Vehicle and Day-to-Day Variability of Traffic Network Travel Times. Transportation Research Part C: Emerging Technologies 46, 8397. doi:10.1016/j.trc.2014.05.011

Kim, J., Mahmassani, H., Vovsha, P., Stogios, Y., Dong, J., 2013b. Scenario-Based Approach to Analysis of Travel Time Reliability with Traffic Simulation Models. Transportation Research Record: Journal of the Transportation Research Board 2391, 56-68. doi:10.3141/2391-06

Lewinski, D., 1983. Nonstationary probabilistic target and clutter scattering models. IEEE Transactions on Antennas and Propagation 31, 490-498. doi:10.1109/TAP.1983.1143067

MacCullagh, P., Nelder, J.A., 1989. Generalized Linear Models. CRC Press.

Mahmassani, H.S., Hou, T., Dong, J., 2012a. Characterizing Travel Time Variability in Vehicular Traffic Networks: Deriving a Robust Relation for Reliability Analysis. Transportation Research Record: Journal of the Transportation Research Board 2315, 141-152. doi:10.3141/2315-15

Mahmassani, H.S., Hou, T., Saberi, M., 2013a. Connecting Networkwide Travel Time Reliability and the Network Fundamental Diagram of Traffic Flow. Transportation Research Record: Journal of the Transportation Research Board 2391, 80-91. doi:10.3141/2391-08

Mahmassani, H.S., Kim, J., Hou, T., Zockaie, A., Saberi, M., Jiang, L., Verbas, O., Cheng, S., Chen, Y., Hass, R., 2012b. Implementation and Evaluation of Weather Responsive Traffic Estimation and Prediction System (Final Report No. FHWA-JPO-12-055). FHWA, U.S. Department of Transportation.

Mahmassani, H.S., Kim, J., Stogios, Y., Currie, K., Vovsha, P., 2013b. Incorporating Reliability Performance Measures in Operations and Planning Modeling Tools (SHRP2 Project L04 Final Report No. S2L04-RR-1).

Mahmassani, H.S., Mudge, R., Hou, T., Kim, J., 2012c. Use of Mobile Data for Weather-Responsive Traffic Management Models (Final Report No. FHWA-JPO-13-003). FHWA, U.S. Department of Transportation.

Mahmassani, H.S., Sbayti, H., 2009. DYNASMART-P Version 1.6 User's Guide.

May, A.D., Bonsall, P.W., Marler, N.W., 1989. Travel Time Variability of a Group of Car Commuters in North London (Monograph No. Working Paper 277). University of Leeds.

Mazloumi, E., Currie, G., Rose, G., 2010. Using GPS Data to Gain Insight into Public Transport Travel Time Variability. Journal of Transportation Engineering 136, 623-631. doi:10.1061/(ASCE)TE.19435436.0000126

Noland, R.B., Polak, J.W., 2002. Travel time variability: A review of theoretical and empirical issues. Transport Reviews 22, 39-54. doi:10.1080/01441640010022456

Peer, S., Koopmans, C.C., Verhoef, E.T., 2012. Prediction of travel time variability for cost-benefit analysis. Transportation Research Part A: Policy and Practice 46, 79-90. doi:10.1016/j.tra.2011.09.016

PeMS [WWW Document], n.d. . Caltrans Performance Measurement System. URL http://pems.dot.ca.gov/ (accessed 4.20.13).

Polus, A., 1979. A study of travel time and reliability on arterial routes. Transportation 8, 141-151. doi:10.1007/BF00167196

$\mathrm{Pu}, \mathrm{W} ., 2011$. Analytic Relationships Between Travel Time Reliability Measures. Transportation Research Record: Journal of the Transportation Research Board 2254, 122-130. doi:10.3141/2254-13

Rakha, H., El-Shawarby, I., Arafeh, M., Dion, F., 2006. Estimating Path Travel-Time Reliability, in: IEEE Intelligent Transportation Systems Conference, 2006. ITSC '06. Presented at the IEEE Intelligent 
Transportation Systems Conference, 2006. ITSC '06, pp. 236-241.

doi:10.1109/ITSC.2006.1706748

Redding, N.J., 1999. Estimating the Parameters of the K Distribution in the Intensity Domain.

Richardson, A.J., Taylor, M., 1978. Travel Time Variability on Commuter Journeys. High Speed Ground Transportation Journal 12.

Shankar, P.M., 2004. Error Rates in Generalized Shadowed Fading Channels. Wireless Personal Communications 28, 233-238. doi:10.1023/B:wire.0000032253.68423.86

Susilawati, S., Taylor, M.A.P., Somenahalli, S.V.C., 2011. Distributions of travel time variability on urban roads. Journal of Advanced Transportation. doi:10.1002/atr.192

Taylor, M., 1982. Travel Time Variability-The Case of Two Public Modes. Transportation Science 16, 507-521. doi:10.1287/trsc.16.4.507

Taylor, M.A.P., Susilawati, 2012. Modelling Travel Time Reliability with the Burr Distribution. Procedia Social and Behavioral Sciences 54, 75-83. doi:10.1016/j.sbspro.2012.09.727

Teich, M.C., Diament, P., 1989. Multiply stochastic representations for K distributions and their Poisson transforms. J. Opt. Soc. Am. A 6, 80-91. doi:10.1364/JOSAA.6.000080

Verbas, I., Mahmassani, H., Zhang, K., 2011. Time-Dependent Origin-Destination Demand Estimation. Transportation Research Record: Journal of the Transportation Research Board 2263, 45-56. doi:10.3141/2263-06

Wang, N., Cheng, J., 2010. Moment-based estimation for the shape parameters of the Gamma-Gamma atmospheric turbulence model. Opt. Express 18, 12824-12831. doi:10.1364/OE.18.012824

Yildirimoglu, M., Koymans, A., Geroliminis, N., 2013. Exploring the Properties of Mean-Variance Relations in Freeway Travel Times, in: Proceedings of the 92nd Annual Meeting of the Transportation Research Board, Washington, D.C. Presented at the Transportation Research Board 92nd Annual Meeting, Washington, D.C. 